\title{
Study on Vibrational Power Flow Propagation Characteristics in a Laminated Composite Cylindrical Shell Filled with Fluid
}

\author{
Jingxi Liu $\left(\mathbb{D},{ }^{1,2}\right.$ Wentao He $\mathbb{D}^{3,4}$ and De Xie ${ }^{1}$ \\ ${ }^{1}$ School of Naval Architecture and Ocean Engineering, Huazhong University of Science and Technology, Wuhan, Hubei 430074, China \\ ${ }^{2}$ Collaborative Innovation Center for Advanced Ship and Deep-Sea Exploration (CISSE), Shanghai 200240, China \\ ${ }^{3}$ College of Engineering, Ocean University of China, Qingdao 266100, China \\ ${ }^{4}$ Shandong Provincial Key Laboratory of Ocean Engineering, Ocean University of China, Qingdao 266100, China
}

Correspondence should be addressed to Wentao He; hewentao@ouc.edu.cn

Received 13 November 2017; Revised 19 April 2018; Accepted 30 April 2018; Published 25 June 2018

Academic Editor: Tai Thai

Copyright (c) 2018 Jingxi Liu et al. This is an open access article distributed under the Creative Commons Attribution License, which permits unrestricted use, distribution, and reproduction in any medium, provided the original work is properly cited.

\begin{abstract}
The characteristics of vibrational power flow in an infinite laminated composite cylindrical shell filled with fluid excited by a circumferential line cosine harmonic force are investigated using wave propagation approach. The harmonic motions of the shell and the fluid filled in the shell are described by Love shell theory and acoustic wave equation, respectively. Under the driving force, the vibrational power flow input into the coupled system and the transmission of the power flow carried by different internal forces (moments) of the shell in the axial direction are established. Numerical computations are implemented to investigate the vibrational power flow input and its propagation. It is found that characteristics of the vibrational power flow vary with different circumferential mode orders and frequencies, and the presence of fluid in the shell significantly affects the vibration of the shell structure. Additionally, parametric investigations are carried out to study the effects of the fiber orientation, modulus ratio $E_{11} / E_{22}$, and thickness-to-radius parameter $h / R$ on input power into the coupled system and propagation power along the shell axial direction. This work will provide some guidance for the vibration control of the laminated composite cylindrical shell.
\end{abstract}

\section{Introduction}

Laminated composite cylindrical shell commonly applied in structural designs is an important element of submerged and floating structures due to its excellent mechanical characteristics of high specific strength-to-weight ratio, good fatigue resistance, and ease of fabrication [1-5]. The vibration problems of fluid-filled shell systems are also common and important in many fields [6-10]. It is well known that vibration not only causes severe noise pollution, but also affects the normal working of equipment installed on piping systems. Therefore, it is very necessary to provide insights into the characteristics of dynamic response for the fluid-filled laminated composite cylindrical shell system.

Numerous researches have been conducted on the vibration characteristics of the composite circular cylindrical shell, and most are focused on vacant shell. For example, Lam et al. [11] investigated the effect of boundary conditions and fiber orientation on the natural frequencies of orthotropic laminated cylindrical shells by Love's first approximation theory and Ritz's procedure. Jafari et al. [12] used the first Love approximation theory to study the free and forced vibration characteristics of the composite circular cylindrical shell. Ferreira et al. [13] employed the first-order theory of Donnell to illuminate the natural frequencies of doubly curved cross-ply composite shells, and a meshless method is adopted for discretization of motion equations. Alibeigloo [14] investigated the vibration characteristics of anisotropic laminated cylindrical shell by using differential quadrature method. Asadi et al. [15] investigated static and free vibration characteristics for cross-ply cylindrical shells by using firstorder shear deformation theory. Viola et al. [16] studied the vibration characteristics of completely doubly curved laminated shells by using a higher-order shear deformation theory, associated with the application of generic shear functions. Khalili et al. [17] studied the free and forced vibration characteristics of the composite cylindrical shell subjected to the transverse impulse loading using the first-order shear 
deformation theory. Amabili and Reddy [18] developed a higher-order shear deformation nonlinear theory, and then large-amplitude forced vibrations of laminated circular cylindrical shell were studied by using the developed theory.

A few investigations have been made on the vibration characteristics of the circular cylindrical shell filled with fluids. Zhang et al. [19] studied the natural frequencies of finite fluid-filled cylindrical shells by using the wave propagation method. The result showed that the fluid effect on the shell was significant. Iqbal et al. [20] used the wave propagation method to make the vibrational analysis of a functionally graded material (FGM) circular cylindrical fluid-filled shell. Natsuki et al. [21] investigated the vibration characteristics of fluid-filled double-walled carbon nanotubes based on the proposed simplified Flügge shell equations using the wave propagation approach. Kadoli and Ganesan [22] studied the free vibrational analysis of the composite cylindrical shell filled with hot fluid based on the first-order shear deformation theory. Paak et al. [23] investigated the large-amplitude vibrations of the circular cylindrical tanks filled with liquid based on Flügge's shell theory. Many studies have been conducted on the vibration of the partially fluid-filled isotropic and composite tanks. Xi et al. [24] studied free vibration of partially fluid-filled laminated composite circular cylindrical shells, and the influence factors such as circumferential wavenumber, fluid depth, and boundary condition have been investigated systemically. Saviz [25] also investigated the free vibration of partially fluid-filled laminated composite circular cylindrical shell based on Rayleigh-Ritz method. Although both vibrational power flow concerning circular cylindrical shell and vibrational characteristics in laminated composite cylindrical shell have been studied by numerous scholars, literatures about the characteristics of vibrational power flow in a laminated composite cylindrical shell filled with fluid are rarely found.

The wave propagation approach is very valuable in the vibrational analysis of thin cylindrical shell. Xu and Zhang [26] studied the characteristics of vibrational power flow in an infinite elastic circular cylindrical shell filled with fluid based on Flügge's thin shell theory and Helmholtz equation. Yan et al. [27] also studied the vibrational power flow for a submerged infinite cylindrical shell excited by a radial cosine harmonic line force based on the wave propagation approach. Gan et al. [28] also studied the free vibration of ring-stiffened cylindrical shell subjected to hydrostatic pressure based on Flügge's classical thin shell theory by the wave propagation approach. Bahrami and Teimourian [29] investigated the free vibration and wave reflection of carbon nanotubes by using the wave propagation approach.

From the above, we can draw the conclusion that much attention has been paid to the free vibration and forced vibration characteristics of the laminated cylindrical shells. Few of them have investigated the vibrational power characteristics of infinite laminated cylindrical shells. In this paper, the characteristics of vibrational power flow in a laminated composite cylindrical shell filled with fluid excited by a circumferential line cosine harmonic force are investigated with wave propagation approach. The harmonic motions of the shell and the fluid filled in the shell are described by Love shell theory and Helmholtz equation, respectively. Vibrational power flow input into the coupled system and its propagation along the shell axial direction are both studied. Additionally, investigations are carried out to study the effects of the fiber orientation, modulus ratio $E_{11} / E_{22}$, and thickness-to-radius parameter $h / R$ on input power into the coupled system and propagation power along the shell axial direction.

\section{Fundamental Equations of Laminated Composite Cylindrical Shell}

The composite cylindrical shell can be defined through thickness $h$, radius $R$, and axial length $L$, as exhibited in Figure 1 (a). The orthogonal coordinate system $(x, \theta$, and $z)$ is built up at the middle surface of the cylindrical shell. The $x, \theta$, and $z$ coordinates are considered as the axial, circumferential, and radial directions of the cylindrical shell, respectively. The corresponding displacement components of the cylindrical shell are expressed as $u, v$, and $w$, respectively.

According to Love theory, the motion equations of the cylindrical shell can be expressed through the force components $N$ and the moment components $M$ :

$$
\begin{array}{r}
\frac{\partial N_{x}}{\partial x}+\frac{\partial N_{x \theta}}{R \partial \theta}=\rho h \frac{\partial^{2} u}{\partial t^{2}} \\
\frac{\partial N_{x \theta}}{\partial x}+\frac{\partial N_{\theta}}{R \partial \theta}+\frac{\partial M_{x \theta}}{R \partial x}+\frac{\partial M_{\theta}}{R^{2} \partial \theta}=\rho h \frac{\partial^{2} v}{\partial t^{2}} \\
\frac{\partial M_{x}}{\partial x^{2}}+\frac{2 \partial^{2} M_{\theta}}{R \partial x \partial \theta}+\frac{\partial^{2} M_{\theta}}{R^{2} \partial \theta^{2}}+\frac{N_{\theta}}{R}=\rho h \frac{\partial^{2} w}{\partial t^{2}}
\end{array}
$$

where $N$ and $M$ can be calculated by the corresponding stress components $\sigma_{x}, \sigma_{\theta}$, and $\sigma_{x \theta}$ at a distance $z$ from the middle surface:

$$
\begin{gathered}
\left(N_{x}, N_{\theta}, N_{x \theta}\right)=\int_{-h / 2}^{h / 2}\left(\sigma_{x}, \sigma_{\theta}, \sigma_{x \theta}\right) d z \\
\left(M_{x}, M_{\theta}, M_{x \theta}\right)=\int_{-h / 2}^{h / 2}\left(\sigma_{x}, \sigma_{\theta}, \sigma_{x \theta}\right) z d z
\end{gathered}
$$

According to Hooke's law, the stress components $\sigma_{x}$, $\sigma_{\theta}$, and $\sigma_{x \theta}$ for a thin shell can be expressed through the corresponding strain components $e_{x}, e_{\theta}$, and $e_{x \theta}$ at a distance $z$ from the middle surface:

$$
\left\{\begin{array}{c}
\sigma_{x} \\
\sigma_{\theta} \\
\sigma_{x \theta}
\end{array}\right\}=\left[\begin{array}{ccc}
Q_{11} & Q_{12} & 0 \\
Q_{12} & Q_{22} & 0 \\
0 & 0 & Q_{66}
\end{array}\right]\left\{\begin{array}{c}
e_{x} \\
e_{\theta} \\
e_{x \theta}
\end{array}\right\}
$$

According to Love's approximation theory, $e_{x}, e_{\theta}$, and $e_{x \theta}$ can be calculated by the middle surface strain components $e_{1}$, $e_{2}$, and $\gamma$ and the thickness coordinate $z$ :

$$
\begin{gathered}
e_{x}=e_{1}+z k_{1} \\
e_{\theta}=e_{2}+z k_{2} \\
e_{x \theta}=\gamma+2 z \tau
\end{gathered}
$$




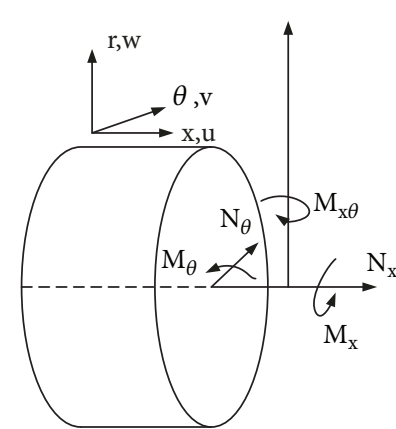

(a)
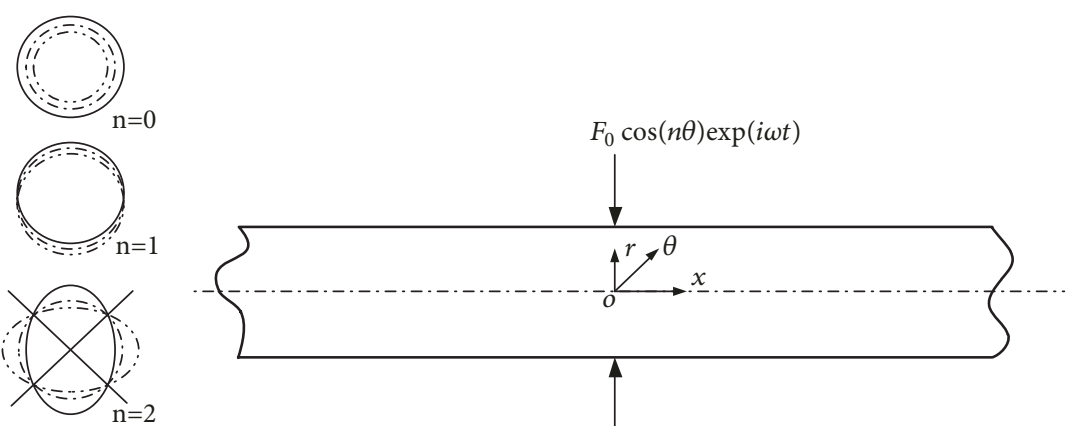

(b)

Figure 1: (a) Coordinate system and circumferential modal shape. (b) Harmonic line force F applied on thin laminated composite cylindrical shell.

where $k_{1}, k_{2}$, and $\tau$ are the middle surface curvature components of the cylindrical shell. The middle surface strain components and their corresponding curvature components are defined according to displacement components of the cylindrical shell:

$$
\begin{aligned}
& \left\{e_{1}, e_{2}, \gamma\right\}=\left\{\frac{\partial u}{\partial x}, \frac{1}{R}\left(\frac{\partial v}{\partial \theta}+w\right), \frac{\partial v}{\partial x}+\frac{1}{R} \frac{\partial u}{\partial \theta}\right\} \\
& \left\{k_{1}, k_{2}, \tau\right\} \\
& =\left\{-\frac{\partial^{2} w}{\partial x^{2}}, \frac{1}{R^{2}}\left(-\frac{\partial^{2} w}{\partial \theta^{2}}+\frac{\partial v}{\partial \theta}\right), \frac{1}{R}\left(-\frac{\partial^{2} w}{\partial x \partial \theta}+\frac{\partial v}{\partial x}\right)\right\}
\end{aligned}
$$

Associated with (2)-(5), the force and moment components can be expressed as

$$
\left\{\begin{array}{c}
N_{x} \\
N_{\theta} \\
N_{x \theta} \\
M_{x} \\
M_{\theta} \\
M_{x \theta}
\end{array}\right\}=\left[\begin{array}{llllll}
A_{11} & A_{12} & A_{16} & B_{11} & B_{12} & B_{16} \\
A_{12} & A_{22} & A_{26} & B_{12} & B_{22} & B_{26} \\
A_{16} & A_{26} & A_{66} & B_{16} & B_{26} & B_{66} \\
B_{11} & B_{12} & B_{16} & D_{11} & D_{12} & D_{16} \\
B_{12} & B_{22} & B_{26} & D_{12} & D_{22} & D_{26} \\
B_{16} & B_{26} & B_{66} & D_{16} & D_{26} & D_{66}
\end{array}\right]\left\{\begin{array}{c}
e_{1} \\
e_{2} \\
\gamma \\
k_{1} \\
k_{2} \\
2 \tau
\end{array}\right\}
$$

where the extensional stiffness $A_{i j}$, coupling stiffness $B_{i j}$, and bending stiffness $D_{i j}$ are expressed as

$$
\begin{aligned}
A_{i j} & =\int_{-h / 2}^{h / 2} \bar{Q}_{i j} d z=\sum_{k=1}^{n} \bar{Q}_{i j}\left(z_{k}-z_{k-1}\right) \\
B_{i j} & =\int_{-h / 2}^{h / 2} z \bar{Q}_{i j} d z=\sum_{k=1}^{n} \bar{Q}_{i j}\left(z^{2}{ }_{k}-z^{2}{ }_{k-1}\right) \\
D_{i j} & =\int_{-h / 2}^{h / 2} z^{2} \bar{Q}_{i j} d z=\sum_{k=1}^{n} \bar{Q}_{i j}\left(z^{3}{ }_{k}-z^{3}{ }_{k-1}\right)
\end{aligned}
$$

where $z_{k-1}$ and $z_{k}$ are the boundaries of the $k^{\text {th }}$ layer. $\bar{Q}_{i j}$ is the elastic stiffness constant of the $k^{\text {th }}$ layer. $\bar{Q}_{i j}$ in (7) is defined as

$$
[\bar{Q}]=[S]^{-1}[Q][S]^{-T}
$$

where the transformation matrix $[S]$ is defined as

$$
[S]=\left[\begin{array}{ccc}
\cos ^{2} \alpha & \sin ^{2} \alpha & 2 \sin \alpha \cos \alpha \\
\sin ^{2} \alpha & \cos ^{2} \alpha & -2 \sin \alpha \cos \alpha \\
-\sin \alpha \cos \alpha & \sin \alpha \cos \alpha & \cos ^{2} \alpha-\sin ^{2} \alpha
\end{array}\right]
$$

For the thin laminated composite cylindrical shell, the modulus components can be obtained from the engineering elasticity constants. The reduced transformed stiffness $Q_{i j}$ can be expressed based on material properties as follows:

$$
\begin{aligned}
Q_{11} & =\frac{E_{11}}{\left(1-\mu_{12} \mu_{21}\right)} \\
Q_{12} & =\frac{E_{22} \mu_{12}}{\left(1-\mu_{12} \mu_{21}\right)} \\
\left(Q_{22}, Q_{66}\right) & =\left(\frac{E_{22}}{\left(1-\mu_{12} \mu_{21}\right)}, G_{12}\right)
\end{aligned}
$$

where $E_{11}$ and $E_{22}$ are longitudinal stiffness and transverse stiffness of the composite shell, respectively; $G_{12}$ is the shear modulus. $\mu_{12}$ and $\mu_{21}$ are Poisson's ratio of the composite shell. Associated with (6)-(10), the motion equation of the composite cylindrical shell can be expressed in a matrix form as

$$
\left[\begin{array}{lll}
T_{11} & T_{12} & T_{13} \\
T_{21} & T_{22} & T_{23} \\
T_{31} & T_{32} & T_{33}
\end{array}\right]\left\{\begin{array}{l}
u \\
v \\
w
\end{array}\right\}=\left\{\begin{array}{l}
0 \\
0 \\
0
\end{array}\right\}
$$




\section{The Response of the Coupled System}

The following spatial displacement field of the cylindrical shell can be expressed in the form of wave propagation:

$$
\begin{gathered}
u=\sum_{n=0}^{\infty} \sum_{m=1}^{\infty} U_{m} \cos (n \theta) \exp \left(i \omega t-i k_{m} x\right) \\
v=\sum_{n=0}^{\infty} \sum_{m=1}^{\infty} V_{m} \sin (n \theta) \exp \left(i \omega t-i k_{m} x\right) \\
w=\sum_{n=0}^{\infty} \sum_{m=1}^{\infty} W_{m} \cos (n \theta) \exp \left(i \omega t-i k_{m} x\right)
\end{gathered}
$$

where $U_{m}, V_{m}$, and $W_{m}$ are constants respecting the amplitudes of displacement in the $x, \theta$, and $z$ directions, respectively. $n$ is the circumferential modal parameter, $\omega$ is the circular driving frequency, and $k_{m}$ is the axial wavenumber.

The fluid in the cylindrical shell is supposed to be incompressible and inviscid which should satisfy the acoustic wave equation. The motion equation of the fluid can be expressed as

$$
\frac{1}{r} \frac{\partial}{\partial r}\left(r \frac{\partial p}{\partial r}\right)+\frac{1}{r^{2}} \frac{\partial^{2} p}{\partial \theta^{2}}+\frac{\partial^{2} p}{\partial x^{2}}=\frac{1}{c^{2}} \frac{\partial^{2} p}{\partial t^{2}}
$$

where $t$ is time, $p$ is the acoustic pressure, and $c$ is the sound speed of the fluid. The associated form of the acoustic pressure field in the coupled system can be defined as

$$
p=\sum_{n=0}^{\infty} \sum_{m=1}^{\infty} p_{m} \cos (n \theta) J_{n}\left(k_{r} r\right) \exp \left(i \omega t-i k_{m} x\right)
$$

where $J_{n}()$ is the Bessel function of circumferential mode order $n$. The parameter $k_{r}$ is the radial wavenumber. Meanwhile, $k_{r}$ and $k_{m}$ should be satisfied by the following equations:

$$
\begin{aligned}
\left(k_{r} R\right)^{2} & =\Omega^{2}\left(\frac{C_{s}}{C_{f}}\right)^{2}-\left(k_{m} R\right)^{2} \\
\Omega & =\omega R \sqrt{\frac{\rho_{s}}{E_{2}}}
\end{aligned}
$$

where $\Omega$ is the nondimensional frequency. $C_{s}$ and $C_{f}$ are the sound speed of the shell and fluid, respectively.

In the vibrational analysis, the radical displacement for the cylindrical shell and filled fluid should be the same at the interface between them to ensure contacting with each other. So the coupled boundary at the interface should meet the following:

$$
-\left.\left\{\frac{1}{\left(i \omega \rho_{f}\right)}\right\}\left(\frac{\partial p}{\partial r}\right)\right|_{r=R}=\left.\left(\frac{\partial w}{\partial t}\right)\right|_{r=R}
$$

and

$$
p_{m}=\left[\frac{\omega^{2} \rho_{f}}{k_{r} J_{n}^{\prime}\left(k_{r} R\right)}\right] W_{m}
$$

where $\rho_{f}$ is the density of the filled fluid and $J_{n}^{\prime}()$ denotes differentiation with respect to the argument $k_{r} R$. Considering the acoustic pressure on the shell and the coupling (14), motion equations of the coupled system can be expressed as

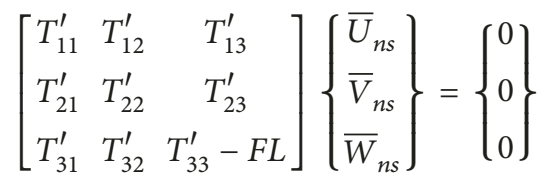

where $T_{i j}^{\prime}(i, j=1,2,3)$ can be obtained by $T_{i j}$ after they are operated on with $x$ and $\theta$.FL is the fluid loading term owing to the acoustic pressure on the shell and is expressed as

$$
F L=\Omega^{2}\left(\frac{\rho_{f}}{\rho_{s}}\right)\left(\frac{R}{h}\right)\left(k_{r} R\right)^{-1}\left[\frac{J_{n}\left(k_{r} R\right)}{J_{n}^{\prime}\left(k_{r} R\right)}\right]
$$

where $\rho_{s}$ is the density of the shell.

\section{Power Flow Input and Transmission}

The shell wall is excited by a harmonic line force $F$ applied on the circumference at $x=0$, and it is specified by

$$
F(\theta, t)=F_{0} \cos (n \theta) \exp (i \omega t)
$$

where the constant term $F_{0}$ represents the amplitude of harmonic line force. The $\operatorname{cosine}$ function $\cos (n \theta)$ represents the distribution of force along circumferential direction. The exponential function $\exp (i \omega t)$ represents the harmonic force. Substituting (12) into the original motion equations of the fluid-filled shell (19) gives the spectral motion equations of the forced response of this coupled system:

$$
\left[\begin{array}{ccc}
T_{11}^{\prime} & T_{12}^{\prime} & T_{13}^{\prime} \\
T_{21}^{\prime} & T_{22}^{\prime} & T_{23}^{\prime} \\
T_{31}^{\prime} & T_{32}^{\prime} & T_{33}^{\prime}-F L
\end{array}\right]\left\{\begin{array}{l}
\bar{U}_{n s} \\
\bar{V}_{n s} \\
\bar{W}_{n s}
\end{array}\right\}=\left\{\begin{array}{c}
0 \\
0 \\
\frac{\Omega^{2} F_{0}}{2 \pi \rho_{s} h \omega^{2}}
\end{array}\right\}
$$

The solutions of the equations are

$$
\left\{\begin{array}{l}
\bar{U}_{n s} \\
\bar{V}_{n s} \\
\bar{W}_{n s}
\end{array}\right\}=\left[\begin{array}{lll}
I_{11} & I_{12} & I_{13} \\
I_{21} & I_{22} & I_{23} \\
I_{31} & I_{32} & I_{33}
\end{array}\right]\left\{\begin{array}{c}
0 \\
0 \\
\frac{\Omega^{2} F_{0}}{2 \pi \rho_{s} h \omega^{2}}
\end{array}\right\}
$$

where the elements of matrix $I_{i j}(i, j=1,2,3)$ are the inverse of matrix $T^{\prime}(i, j=1,2,3)$ and can be given as

$$
\begin{aligned}
& I_{13}=\frac{\left(T_{12}^{\prime} T_{23}^{\prime}-T_{13}^{\prime} T_{22}^{\prime}\right)}{\left(\operatorname{det}\left|T^{\prime}\right|\right)} \\
& I_{23}=\frac{\left(T_{12}^{\prime} T_{13}^{\prime}-T_{11}^{\prime} T_{32}^{\prime}\right)}{\left(\operatorname{det}\left|T^{\prime}\right|\right)} \\
& I_{33}=\frac{\left(T_{11}^{\prime} T_{22}^{\prime}-T_{12}^{\prime} T_{21}^{\prime}\right)}{\left(\operatorname{det}\left|T^{\prime}\right|\right)}
\end{aligned}
$$


Through making the inverse Fourier transform of (23), the shell displacements can be obtained as

$$
\begin{aligned}
& {\left[\begin{array}{c}
u(x) \\
v(x) \\
w(x)
\end{array}\right]=\frac{\Omega^{2} F_{0}}{2 \pi \rho_{s} h \omega^{2}}} \\
& \quad\left[\begin{array}{l}
\int_{-\infty}^{\infty} I_{13} \cos (n \theta) \exp \left(i \omega t-i k_{n s} x\right) \mathrm{d} k_{n s} \\
\int_{-\infty}^{\infty} I_{23} \sin (n \theta) \exp \left(i \omega t-i k_{n s} x\right) \mathrm{d} k_{n s} \\
\int_{-\infty}^{\infty} I_{33} \cos (n \theta) \exp \left(i \omega t-i k_{n s} x\right) \mathrm{d} k_{n s}
\end{array}\right]
\end{aligned}
$$

When the shell is excited by an external harmonic force $F(\theta, t)$ radially, the radial response of the shell at the cross section $x=0$ can be calculated according to (20) and (24). Therefore, the input power flow into the shell produced by the exciting force can be obtained as

$$
\begin{aligned}
P_{\text {input }} & =\int_{0}^{2 \pi} \frac{1}{2} \operatorname{Re}\left[F_{0} \cos (n \theta) \frac{\partial w(0)^{*}}{\partial t}\right] R \mathrm{~d} \theta \\
& =\frac{\pi}{2 \eta_{n}} \operatorname{Re}\left[i \omega F_{0} w(0)\right]
\end{aligned}
$$

Here, the asterisk represents the complex conjugate, and

$$
\eta_{n}= \begin{cases}0.5 & n=0 \\ 1 & n \neq 0\end{cases}
$$

And the nondimensional input power flow can be expressed as

$$
P_{\text {input }}^{\prime}=\frac{P_{\text {input }}}{F_{0}^{2} \pi} \sqrt{\rho_{s} E_{2} R^{2}}
$$

When the shell wall is subjected to the radial exciting force, the vibrational input power flow will be transmitted in the axial direction from the exciting location, along with the generated vibration waves propagating. The displacement components $u(x), v(x)$, and $w(x)$ of the shell and its radial slope $\partial w(x) / \partial x$ at $x=0$ can be obtained from (24). There are four internal forces (moments) of the shell wall in the axial direction, which are the axial force $\left(N_{x}\right)$, torsional shear force $\left(T_{x}\right)$, transverse shear force $\left(S_{x}\right)$, and bending moment $\left(M_{x}\right)$, respectively. Associated with displacement components, the components of vibrational power flow carried by the internal forces (moments) at the cross section $x=L$ can be obtained as

$$
\begin{aligned}
P_{N x} & =\frac{1}{2 \eta_{n}} \pi \operatorname{Re}\left[i \omega N_{N x} U_{N x}^{*}\right] \\
P_{T x} & =\frac{1}{2 \eta_{n}} \pi \operatorname{Re}\left[i \omega T_{N x} V_{N x}^{*}\right] \\
P_{S x} & =\frac{1}{2 \eta_{n}} \pi \operatorname{Re}\left[i \omega S_{N x} W_{N x}^{*}\right] \\
P_{M x} & =\frac{1}{2 \eta_{n}} \pi \operatorname{Re}\left[i \omega M_{N x}\left(\frac{\partial w}{\partial x}\right)^{*}\right]
\end{aligned}
$$

The total vibrational power flow can be written as

$$
P_{\text {shell }}=P_{N x}+P_{T x}+P_{S x}+P_{M x}
$$

Once the driving force inputs power flow into the shellfluid coupled system, the four kinds of power flow will be transmitted in the axial direction from the exciting location. Due to symmetry of the transmission, only half of the input power will be considered in the positive direction of the shell. From the viewpoint of energy, four kinds of power flow can be characterized by the ratios of the power flow carried by different shell internal forces (moment) to the total power in the shell wall, namely, $P_{N(x)}^{\prime}=P_{N x} / P_{\text {shell }}, P_{T(x)}^{\prime}=P_{T x} / P_{\text {shell }}$, $P_{S(x)}^{\prime}=P_{S x} / P_{\text {shell }}$, and $P_{M(x)}^{\prime}=P_{M x} / P_{\text {shell }}$. The power flow components $P_{N(x)}^{\prime}, P_{T(x)}^{\prime}$, and $P_{S M(x)}^{\prime}=P_{S(x)}^{\prime}+P_{M(x)}^{\prime}$ denote the shell motion in the extensional, torsional, and radial directions, respectively.

\section{Verification of the Present Method}

In this paper, a simple numerical method [27] is employed to obtain the integral in (22). It should be mentioned that structural damping is adopted to shift Young's modules $E_{2}$ to be complex, namely, $E_{2}^{\prime}=E_{2}(1-i \xi)$, which can avoid singularities occurring in obtaining the integral. It is necessary to determine the appropriate upper truncation point in integral ranges. If the error between the integral values in $[-b, b]$ and in $[-0.5 b, 0.5 b]$ is not more than $1 \%$, the parameter $b$ is considered as the upper truncation point. And then the exact integral value can be calculated by Gauss integral method in this integral range.

To check the accuracy of the uncoupled vibration (the case of $F L=0$ ) analysis of a finite shell, the nondimensional frequency parameters $\Omega=\omega R \sqrt{\rho_{s} / E_{22}}$ are compared with those in the literatures [30,31], as shown in Table 1. Here, $E_{22}$ is Young's modulus of the laminated shell. The natural frequencies of the three-layered cross-ply $\left[0^{\circ} / 90^{\circ} / 0^{\circ}\right]$ orthotropic cylindrical shell wall with a simply supportedsimply supported (SS-SS) boundary condition are investigated. For the SS-SS boundary condition of the beam, the wavenumber equation is $k L=m \pi$. The material parameters of the shell are given as $E_{22}=7.6 \mathrm{GN} / \mathrm{m}^{2}, E_{11} / E_{22}=2.5$, $\mu_{1}=0.25$, and $\rho=1640 \mathrm{~kg} / \mathrm{m}^{3}$. The comparisons are presented for the geometric ratios $h / R=0.002$, and $L / R=10.0$ and 20.0. Here, a value of $m=1$ is adopted and corresponding parameter $n$ is selected from 1 to 10 for these comparisons. The comparisons demonstrate that there is a good agreement between the results of the present analysis and [30, 31].

To check the accuracy of the coupled vibration (the case of FL $\neq 0$ ) analysis of a finite shell, no available results for the laminated cylindrical shell are found in the published literature. Therefore, the isotropic cylindrical shell with clamped-clamped (C-C) boundary condition is chosen to verify the proposed coupled vibration analysis, and the natural frequencies are compared with those in the literature [19]. The shell is made of steel with mass density $\rho_{1}=$ $7850 \mathrm{~kg} / \mathrm{m}^{3}$, Poisson's ratio $v=0.3$, and Young's modulus $E=$ $210 \mathrm{GPa}$. The length, radius, and thickness of the shell wall are, respectively, 20,1, and $0.01 \mathrm{~m}$. The shell is filled with water 
TABLE 1: Comparison of the frequency parameters for a three-layered, cross-ply $\left[0^{\circ} / 90^{\circ} / 0^{\circ}\right]$ cylindrical shell with SS boundary conditions $(h / R=0.002, F L=0)$.

\begin{tabular}{ccccc}
\hline$L / R$ & $\mathrm{n}$ & Lam $[30]$ & Zhang[31] & Present \\
\hline \multirow{4}{*}{10} & 1 & 0.083908 & 0.083908 & 0.083908 \\
& 2 & 0.030009 & 0.030008 & 0.030009 \\
& 3 & 0.015193 & 0.015191 & 0.015193 \\
& 4 & 0.012176 & 0.012174 & 0.012176 \\
& 5 & 0.015231 & 0.015230 & 0.015231 \\
& 6 & 0.021179 & 0.021178 & 0.021179 \\
\hline \multirow{3}{*}{20} & 1 & 0.023590 & 0.023589 & 0.023590 \\
& 2 & 0.007904 & 0.007903 & 0.007904 \\
& 3 & 0.005869 & 0.005868 & 0.005869 \\
& 4 & 0.009020 & 0.009019 & 0.009020 \\
& 5 & 0.014236 & 0.014235 & 0.014236 \\
& 6 & 0.020801 & 0.020801 & 0 \\
\hline
\end{tabular}
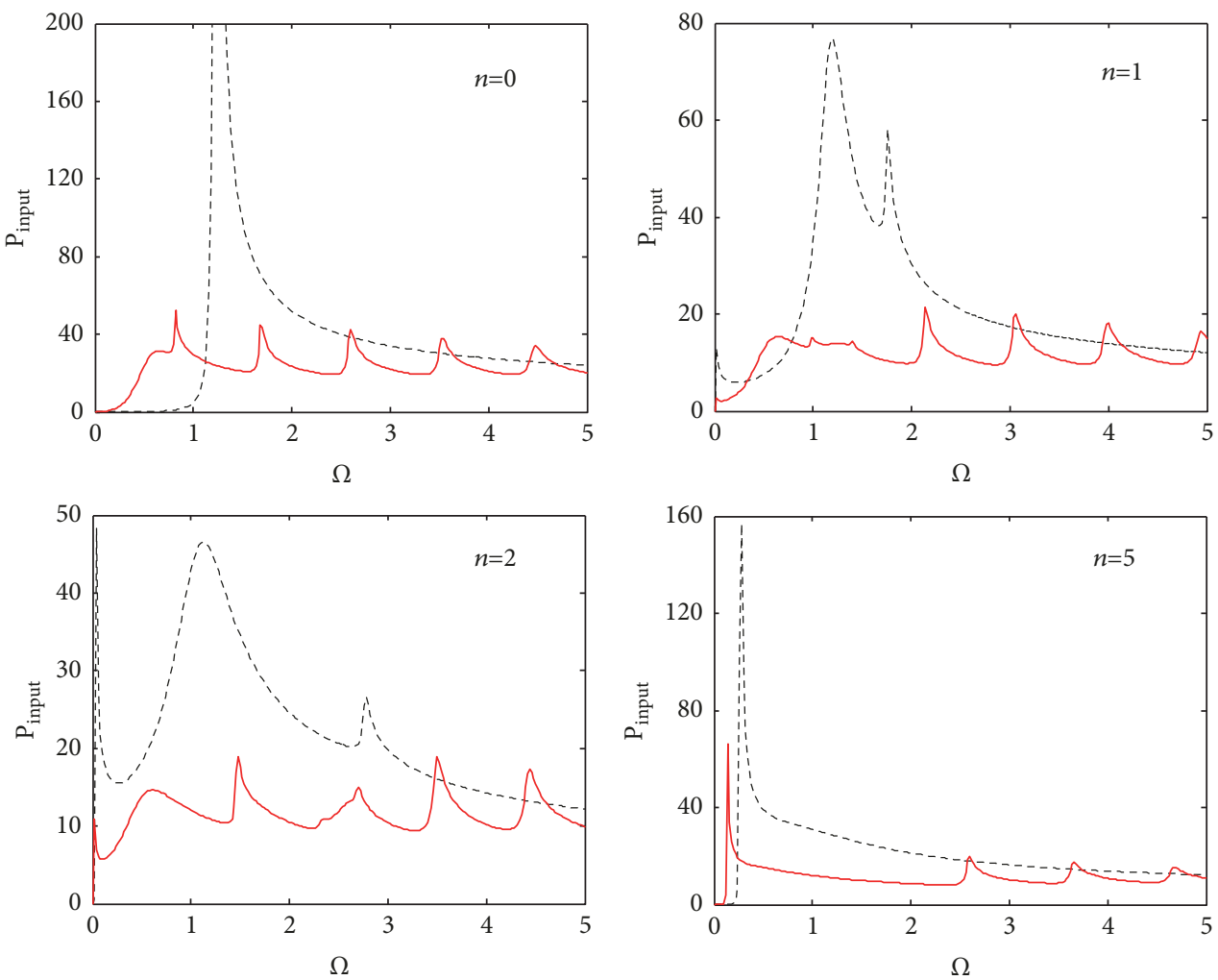

FIGURE 2: The comparison of nondimensional input power flow into the shell filled and unfilled with water. Black dashed line: in vacuo; red solid line: water-filled.

whose sound speed $c=1500 \mathrm{~m} / \mathrm{s}$ and mass density $\rho_{2}=1000$ $\mathrm{kg} / \mathrm{m}^{3}$. The modal vector of the coupled vibration is defined as mode of the axial wavenumber $m$ and the circumferential wavenumber $n$ of standing wave. The first eight coupled natural frequencies of the shell can be obtained by the abovementioned analysis and compared with the results obtained by the FEM/BEM (2000 and 2800 elements) analysis with SYSNOISE, as shown in Table 2. The differences between the present analysis and [19] are within 3.5\%. The comparisons indicate that the proposed coupled vibration analysis method is reasonable and the calculated results are reliable.

\section{Numerical Results and Discussion}

Some numerical computations are conducted to investigate the vibration analysis of the coupled system, including the power flow input into the shell wall and its power flow transmission in the axial direction. Besides, the influence factors such as the fiber orientation, modulus ratio $E_{11} / E_{22}$, and thickness-to-radius parameter $h / R$ are discussed in detail. The following parameters of the coupled system have been employed in the analysis. The material parameters of the shell are given as $E_{22}=7.6 \mathrm{GN} / \mathrm{m}^{2}, E_{11} / E_{22}=2.5, \mu_{1}=0.25$, 


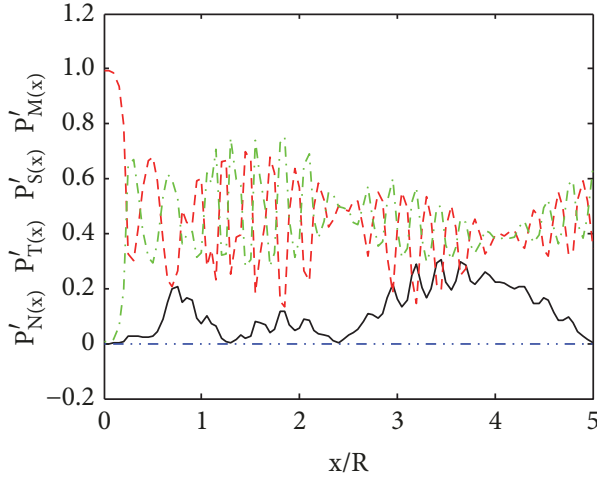

Filled-water

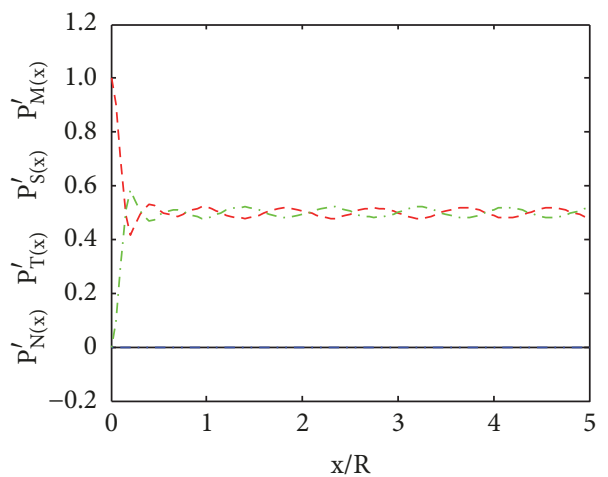

Filled-water

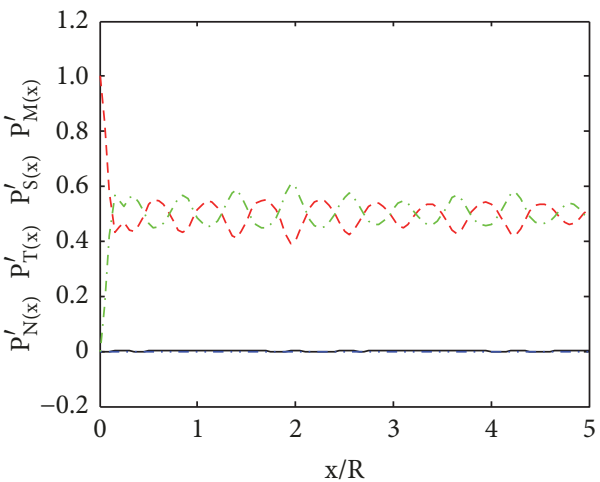

Filled-water

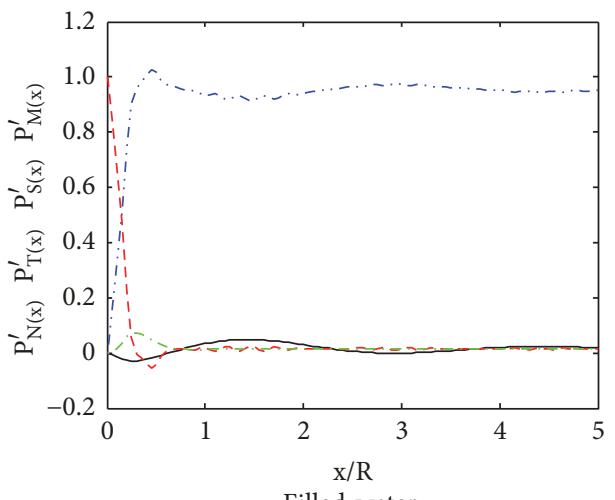

Filled-water

b)

(c)

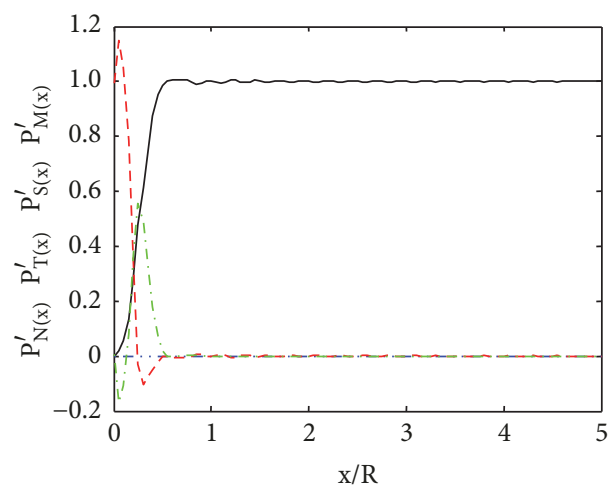

In vacuo

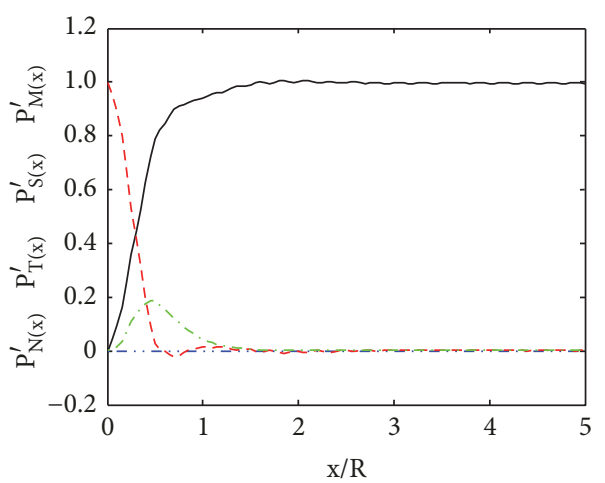

In vacuo

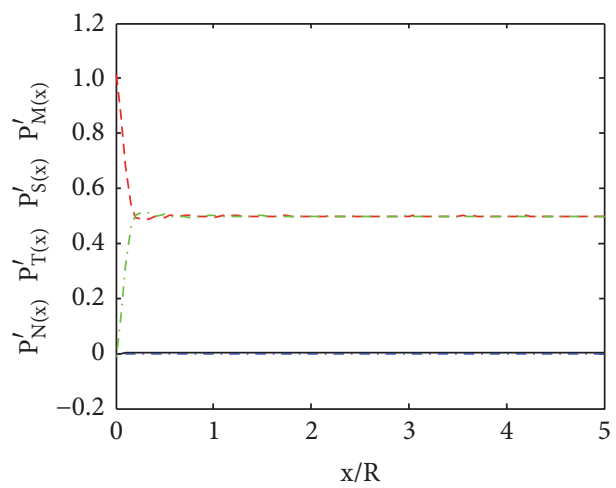

In vacuo

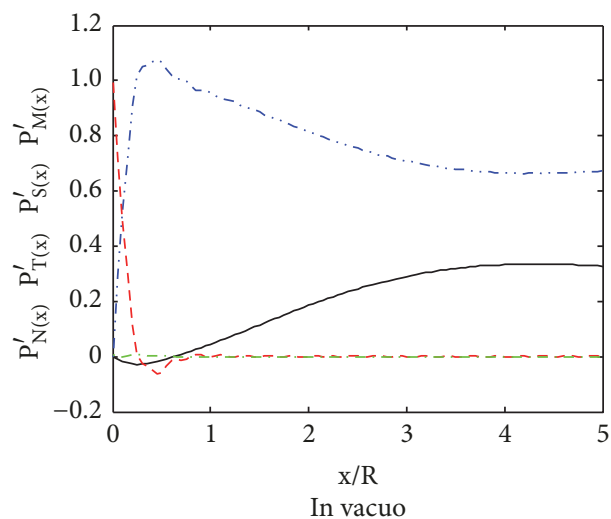

(d)

Figure 3: Continued. 


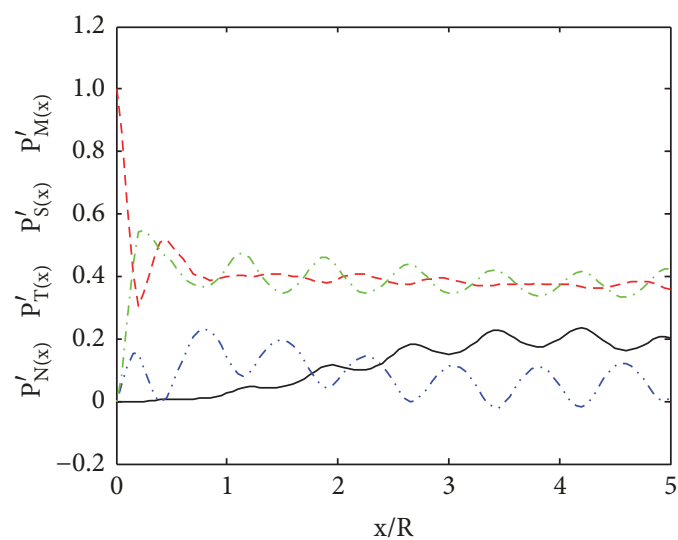

Filled-water

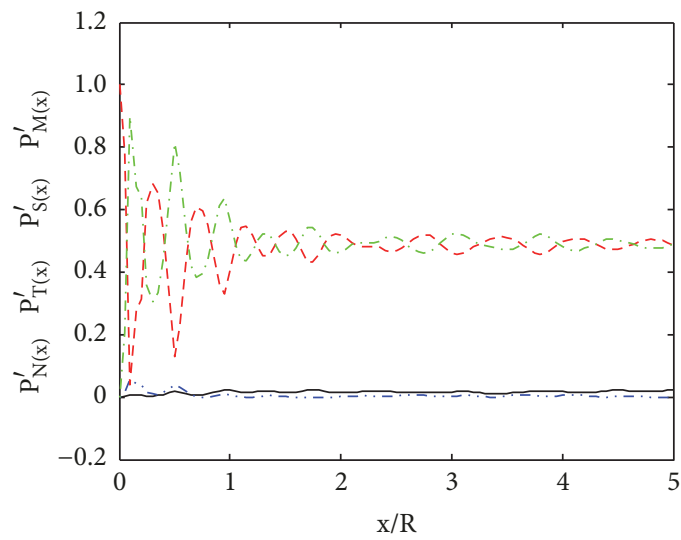

Filled-water

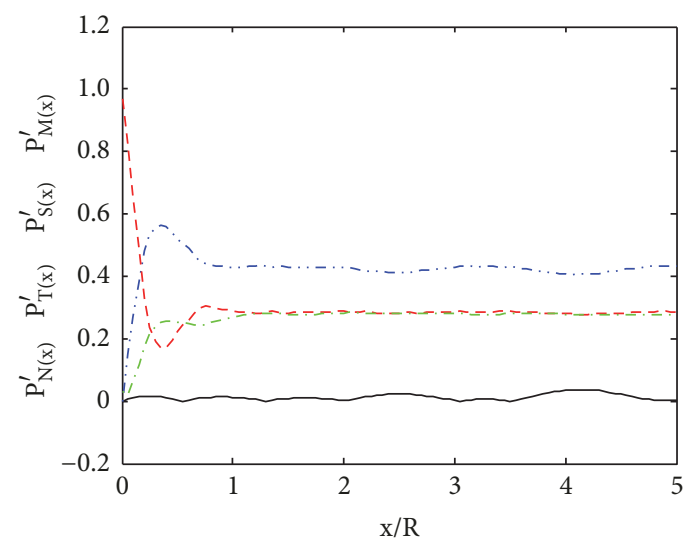

In vacuo

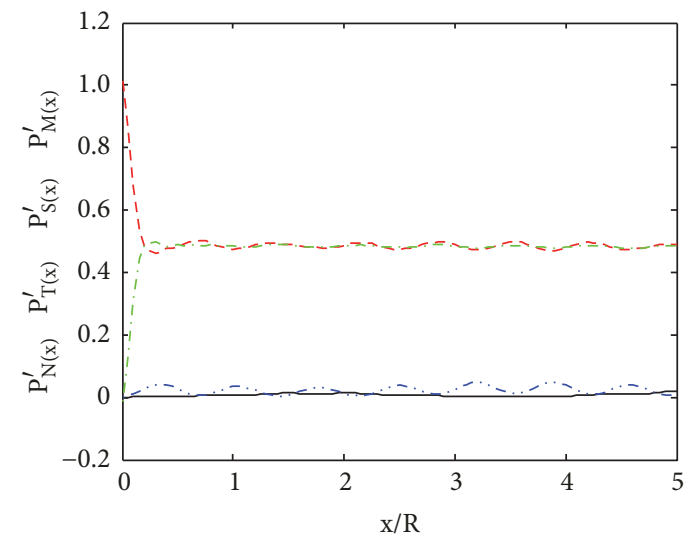

In vacuo

(f)

Figure 3: Power transmitted by different shell forces (moments) for shell filled and unfilled with water: (a) $n=0, \Omega=0.3$; (b) $n=0, \Omega=1.2$; (c) $n=0, \Omega=3.0$; (d) $n=1, \Omega=0.3$; (e) $n=1, \Omega=1.2$; (f) $n=1, \Omega=3.0$; black solid line: $P_{S(x)}^{\prime}$; blue dashed double dotted line: $P_{T(x)}^{\prime}$; red dashed line: $P_{S(x)}^{\prime}$; green dashed dotted line: $P_{M(x)}^{\prime}$.

TABLE 2: Comparison of frequency for a C-C cylindrical shell ( $L=20 \mathrm{~m}, R=1 \mathrm{~m}$, and $h=0.01 \mathrm{~m})$.

\begin{tabular}{|c|c|c|c|c|c|}
\hline \multicolumn{6}{|c|}{ Frequency (HZ) } \\
\hline Order & Modal vector $(\mathrm{m}, \mathrm{n})$ & FEM/BEM(2000) & FEM/BEM $(2800)$ & Reference [19] & present \\
\hline 1 & 1,2 & 4.91 & 4.89 & 4.93 & 4.92 \\
\hline 2 & 1,3 & 9.13 & 9.00 & 8.94 & 8.91 \\
\hline 3 & 2,3 & 10.8 & 10.64 & 10.64 & 10.61 \\
\hline 4 & 2,2 & 11.19 & 11.12 & 11.48 & 11.18 \\
\hline 5 & 3,3 & 14.79 & 14.55 & 14.66 & 14.32 \\
\hline 6 & 1,4 & 18.99 & 18.55 & 18.26 & 18.37 \\
\hline 7 & 2,4 & 19.46 & 19.00 & 18.73 & 19.22 \\
\hline 8 & 3,4 & 20.7 & 20.21 & 19.96 & 20.18 \\
\hline
\end{tabular}

$\rho=1640 \mathrm{~kg} / \mathrm{m}^{3}$, and $h / R=0.0375$. The shell with cross-ply $\left[0^{\circ} / 90^{\circ} / 0^{\circ}\right]$ is filled with water of sound speed $c=1500 \mathrm{~m} / \mathrm{s}$ and mass density $\rho_{2}=1000 \mathrm{~kg} / \mathrm{m}^{3}$. The magnitude of radial force is supposed to be $F=1 \mathrm{~N}$.

6.1. The Vibrational Power Flow Analysis between Filled Fluid and In Vacuo Shell. The nondimensional input flow $P_{\text {input }}^{\prime}$ against the nondimensional driving frequency $\Omega$ for fluidfilled cylindrical shell of different circumferential mode order $n$ is plotted in Figure 2. In order to investigate the effect of filled water, the results of the same shell in vacuo are also plotted. It is obvious that the input power flow varies with circumferential mode order and nondimensional frequency. (1) Due to existing fluid, there are many peaks in curve 

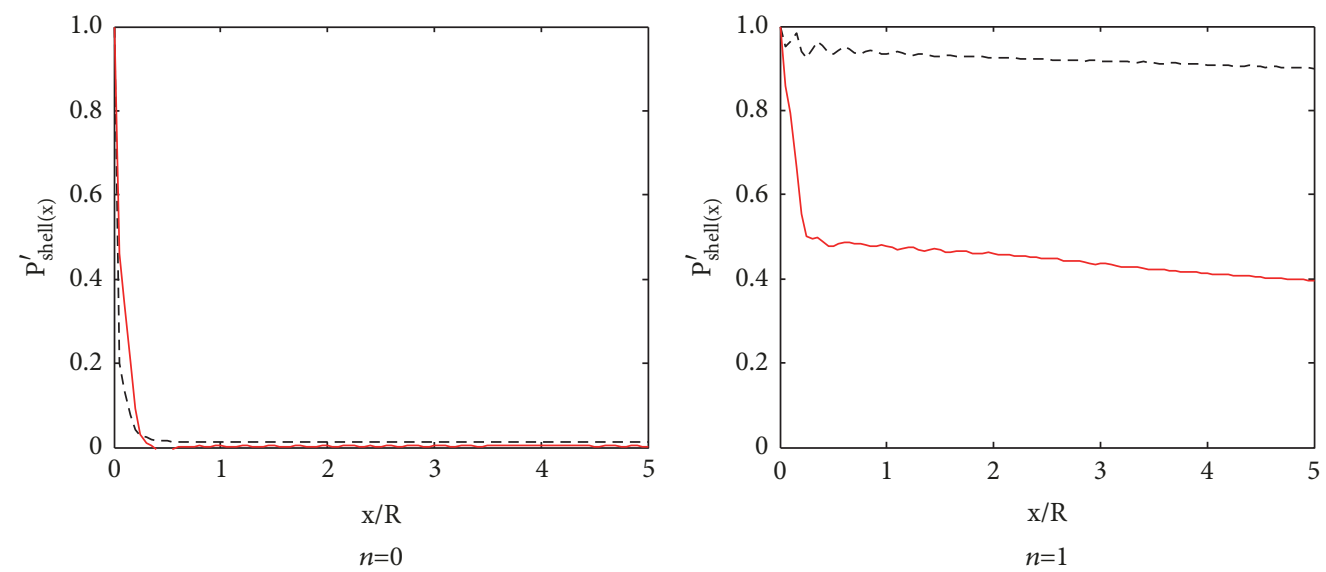

(a)
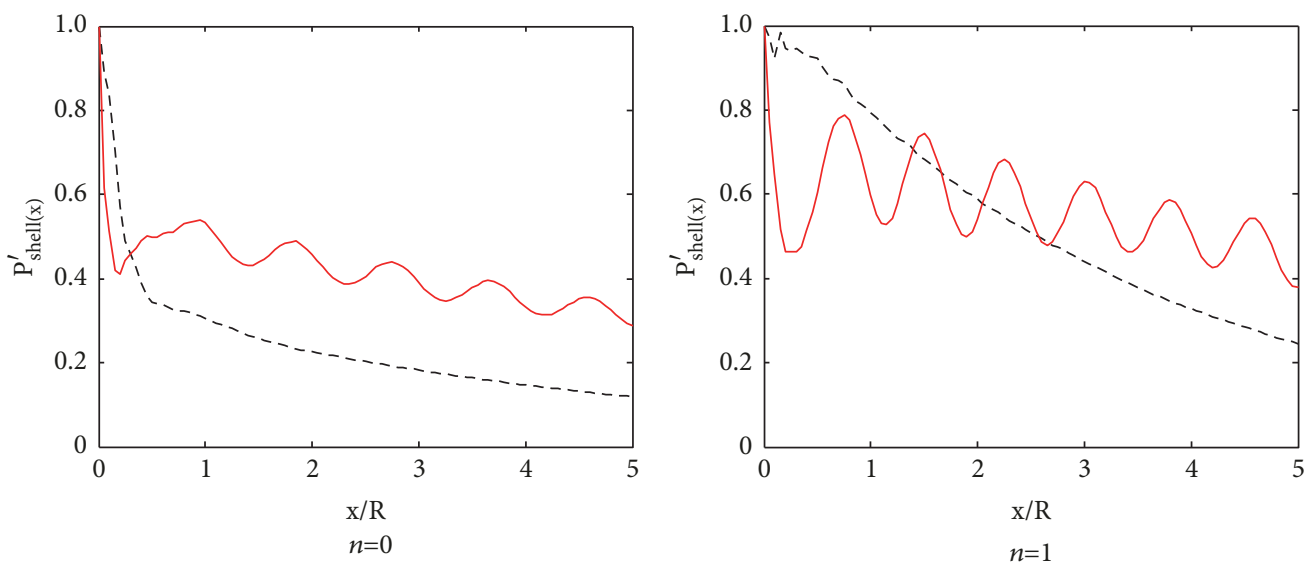

(b)
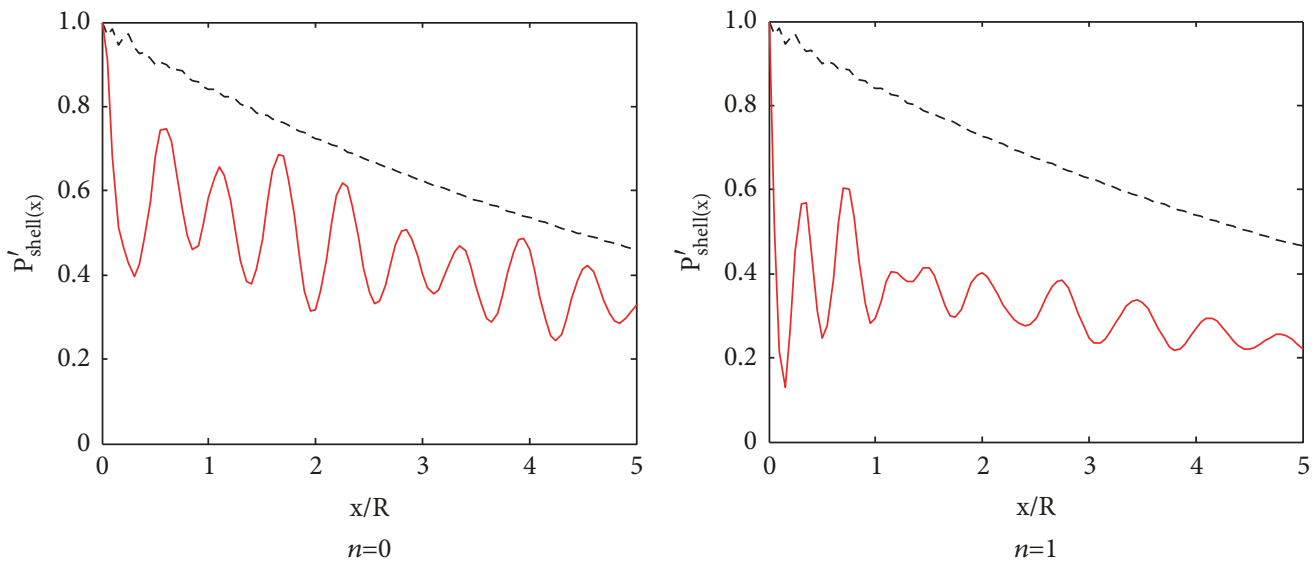

(c)

FIgURe 4: Power transmitted by the shell filled and unfilled with water: (a) $\Omega=0.3$, (b) $\Omega=1.2$, and (c) $\Omega=3.0$; black dashed line: in vacuo; red solid line: water-filled.

of input power flow for a fluid-filled shell compared with shell in vacuo. (2) When $\Omega<3.0$, the input power flow for a shell in vacuo is much larger than that for a fluid-filled shell. The reason is that the shell in vacuo vibrates resonantly with the external force, while the fluid contained in the shell has the effect of reducing the resonant response. But with the frequency increasing, the difference in input power between fluid-filled shell and a shell in vacuo is negligible. The reason is that the coupling effect is weakening with frequency increasing. (3) With the increasing of the circumferential mode order, the peak amplitude of input power flow of shellfluid coupled system shows a decrease while it increases when $n=5$. The reason is that the increasing of circumferential modal parameter is beneficial to the absorption of external 

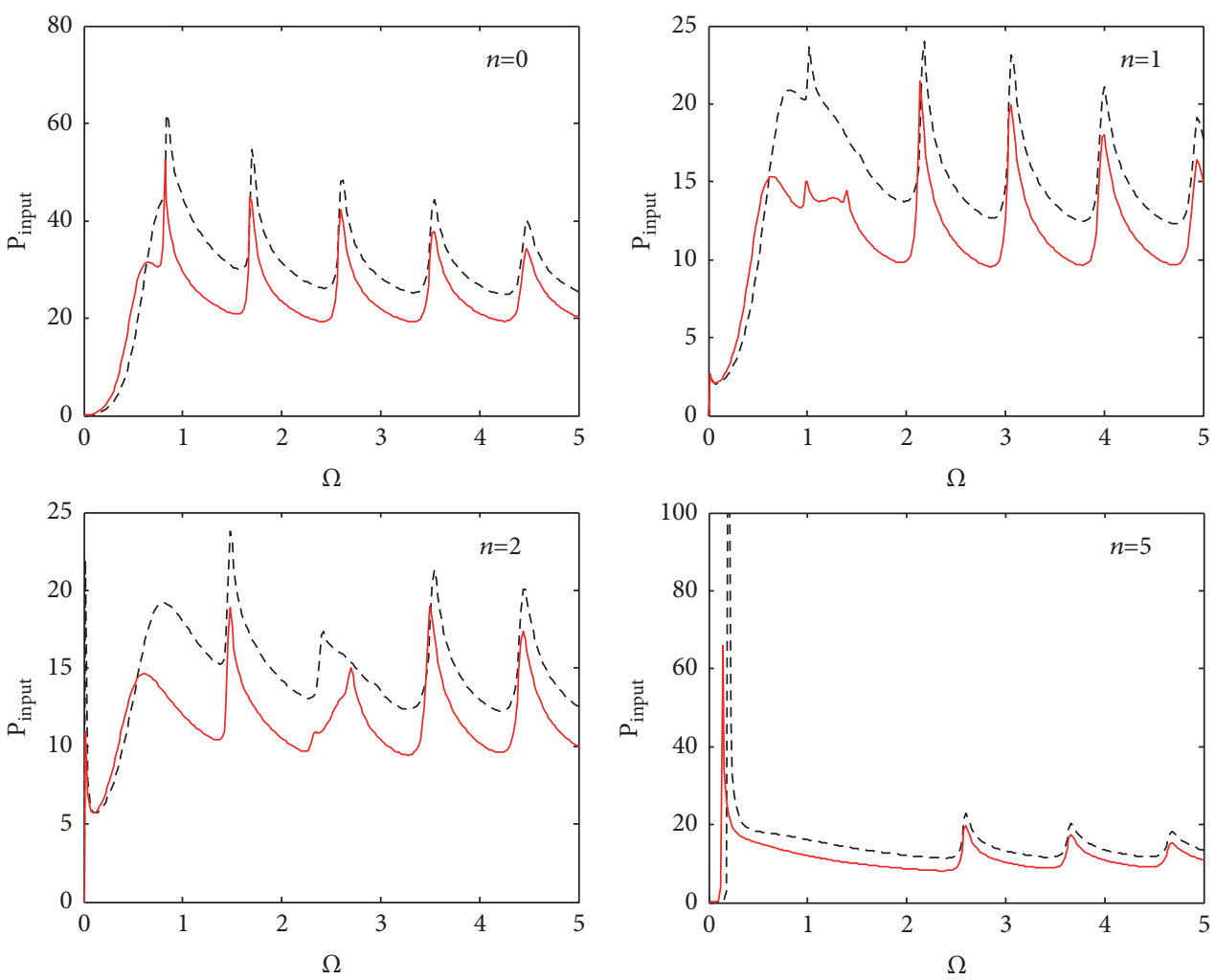

Figure 5: The comparison of nondimensional input power flow into the shell with different cross-ply: black dashed line: [ $90^{\circ} / 0^{\circ} / 90^{\circ}$ ]; red solid line: $\left[0^{\circ} / 90^{\circ} / 0^{\circ}\right]$.

force power flow. From the above, it can be concluded that the filled water will greatly influence the input power flow as a result of an additional mass of water and the shell-fluid interaction at middle frequencies.

The power flow carried by different internal forces (moment) $P_{N(x)}^{\prime}, P_{T(x)}^{\prime}, P_{S(x)}^{\prime}$, and $P_{M(x)}^{\prime}$ of the fluid-filled shell and vacant shell against the nondimensional axial distance $x / R$ is investigated, as shown in Figure 3. The low circumferential mode orders $n=0$ and $n=1$ are discussed in the following, which are the most common cases for the coupled system. Correspondingly, three kinds of representative frequencies (low frequency $\Omega=0.3$, middle frequency $\Omega=1.2$, and high frequency $\Omega=3.0$ ) are chosen for analysis. It should be noted that the negative values represent the notion that the power flow may be transmitted in the opposite direction. At the driving point $x / R=0$, there is $P_{S(x)}^{\prime}=1$ at any frequency for any circumferential mode order. In other words, at the vicinity of the driving point, the total energy transmitted by the shell is almost concentrated on flexural motion; as $x / R$ increases, the flexural motion weakens rapidly, and other motion modes appear. For the in vacuo shell, $P_{S(x)}^{\prime}+$ $P_{M(x)}^{\prime}<P_{N(x)}^{\prime}+P_{T(x)}^{\prime}$ at low frequencies, the power is mainly transmitted by axial force and torsional shear force, whereas the ratio of the power $P_{S(x)}^{\prime}+P_{M(x)}^{\prime}$ increases as circumferential mode order increases. However, $P_{S(x)}^{\prime}+P_{M(x)}^{\prime}>P_{N(x)}^{\prime}+P_{T(x)}^{\prime}$ at high frequencies for any circumferential mode order; this implies that the motion of the shell wall is mainly in radial direction and the power is transmitted by the transverse shear force and bending moment. Similar phenomena can be found for the vacant shell. Besides, for the shell with filled water, $P_{S(x)}^{\prime} \approx P_{M(x)}^{\prime}$ at middle frequencies and high frequencies for any circumferential mode order, while $P_{S(x)}^{\prime} \approx P_{M(x)}^{\prime}$ at high frequencies for the in vacuo shell. This means that the propagation power carried by the transverse shear force is almost equal to that carried by the bending moments.

The power flow $P_{\text {shell }(x)}^{\prime}$ transmitted by the shell in the coupled system against axial distance $x / R$ is described in Figure 4. For comparison, the power flow transmitted by in vacuo shell is also plotted. The investigation also focuses on three typical frequencies for $n=0$ and $n=1$. As $x / R$ increases, the shell wall experiences interaction with filled water, and $P_{\text {shell }(x)}^{\prime}$ attenuates gradually along the shell axial direction due to the energy dissipation of damping and sound radiation. The decay speed of fluid-filled shell is much quicker than that of vacant shell at the vicinity of the driving point. The attenuation of vacant shell is nearly linear, while the attenuation of fluid-filled shell has a periodic wave motion, which means the shell-fluid coupling occurs. The power flow transmitted by the shell filled with water is significantly smaller than that by the in vacuo shell, except for the middle frequency. At the middle frequency, the attenuation of the vibrational power flow transmitted by the shell filled with water is quicker than those by in vacuo shell at the driving point, while being gradually slower than those by in vacuo 

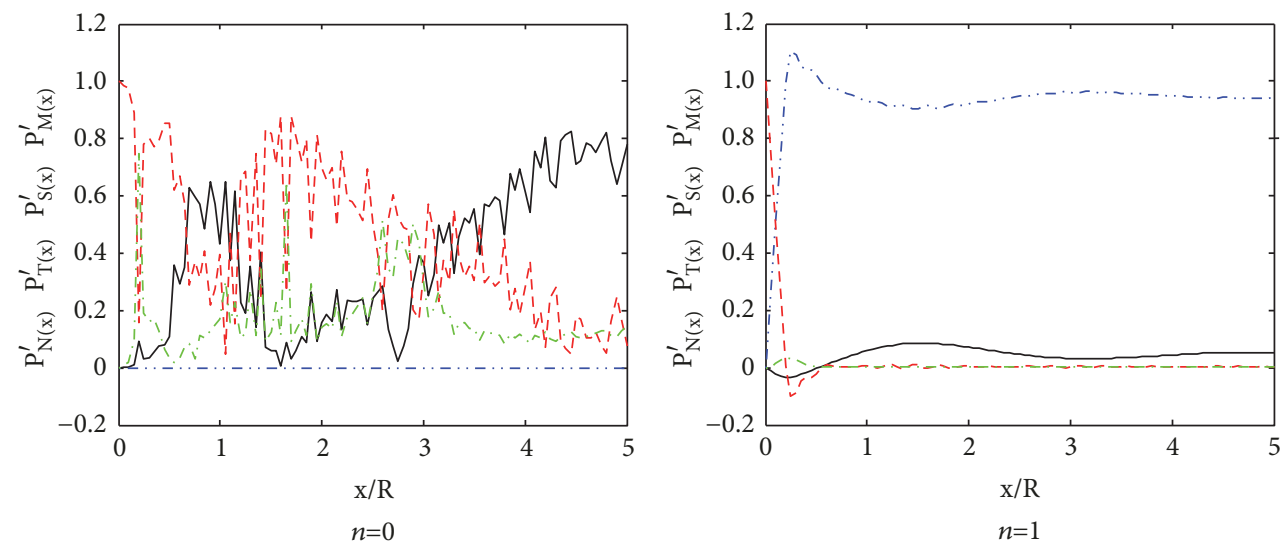

(a)
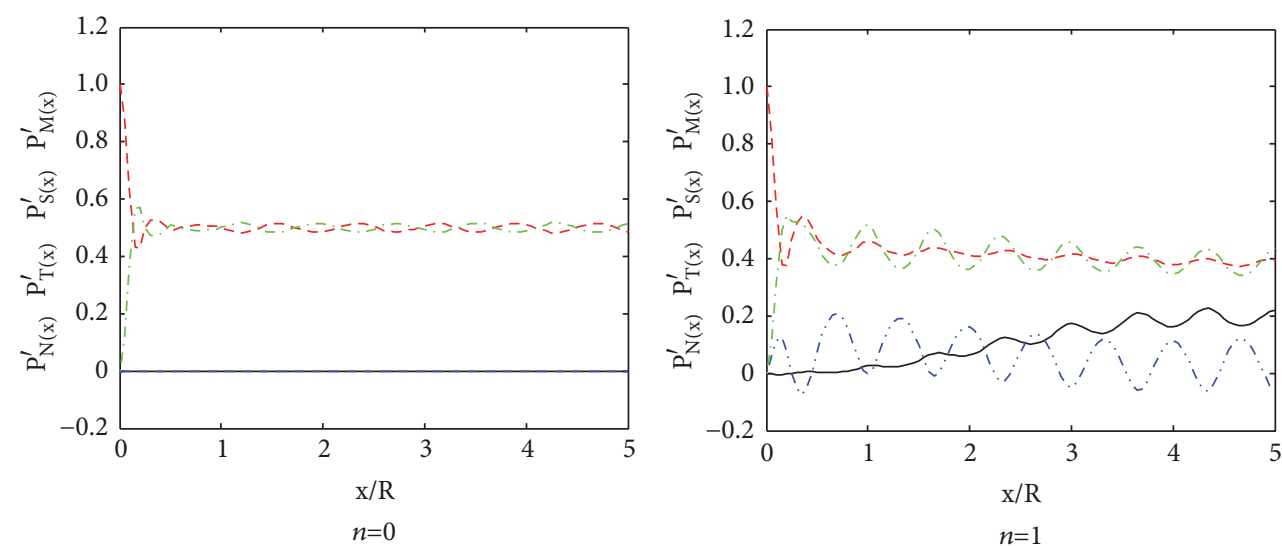

(b)
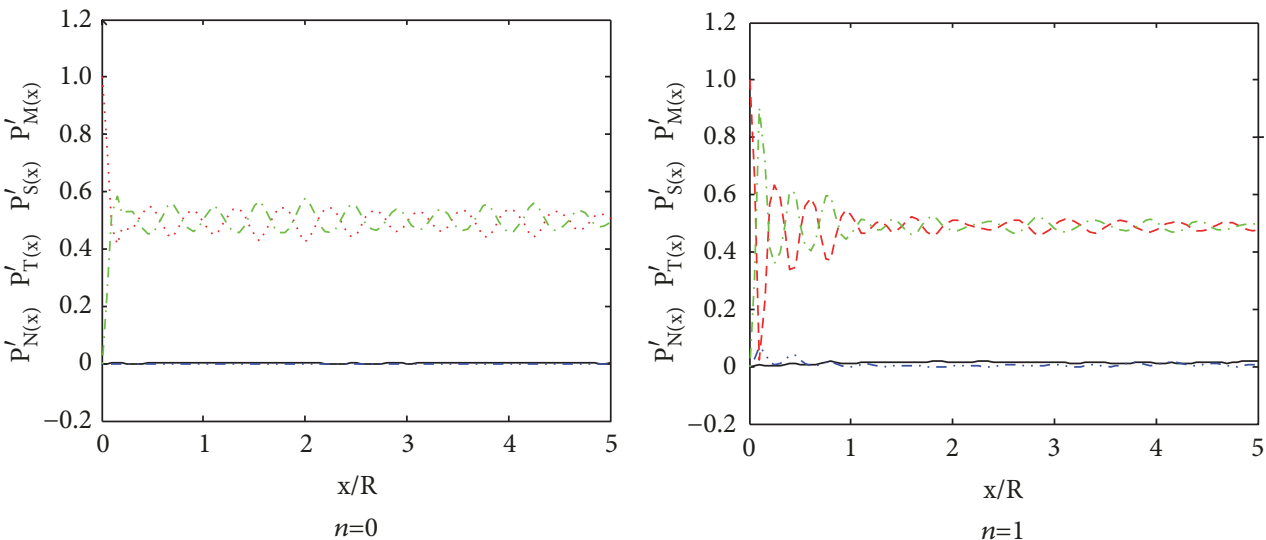

(c)

Figure 6: Power transmitted by different shell forces (moments) with cross-ply [ $90^{\circ} / 0^{\circ} / 90^{\circ}$ ]: (a) $\Omega=0.3$, (b) $\Omega=1.2$, and (c) $\Omega=3.0$; black solid line: $P_{S(x)}^{\prime}$; blue dashed double dotted line: $P_{T(x)}^{\prime}$; red dashed line: $P_{S(x)}^{\prime}$; green dashed dotted line: $P_{M(x)}^{\prime}$.

shell along the axial direction. Thus, it can be concluded that the existence of fluid in the shell significantly affects the vibration characteristics of the shell.

6.2. The Influence of Fiber Orientation. In order to illuminate the influence of layer orientation of fiber on the vibration analysis, the fluid-filled cylindrical shell with cross-ply $\left[90^{\circ} / 0^{\circ} / 90^{\circ}\right]$ is chosen to compare with the original model. The input flow $P_{\text {input }}^{\prime}$ against the driving frequency $\Omega$ for fluid-filled cylindrical shell with cross-ply $\left[90^{\circ} / 0^{\circ} / 90^{\circ}\right]$ and $\left[0^{\circ} / 90^{\circ} / 0^{\circ}\right]$ is plotted in Figure 5. The profile of the input power flow into the cross-ply $\left[90^{\circ} / 0^{\circ} / 90^{\circ}\right]$ shell is similar to that into the cross-ply $\left[0^{\circ} / 90^{\circ} / 0^{\circ}\right]$ shell, while the former becomes clearly bigger than the latter. This is because the 

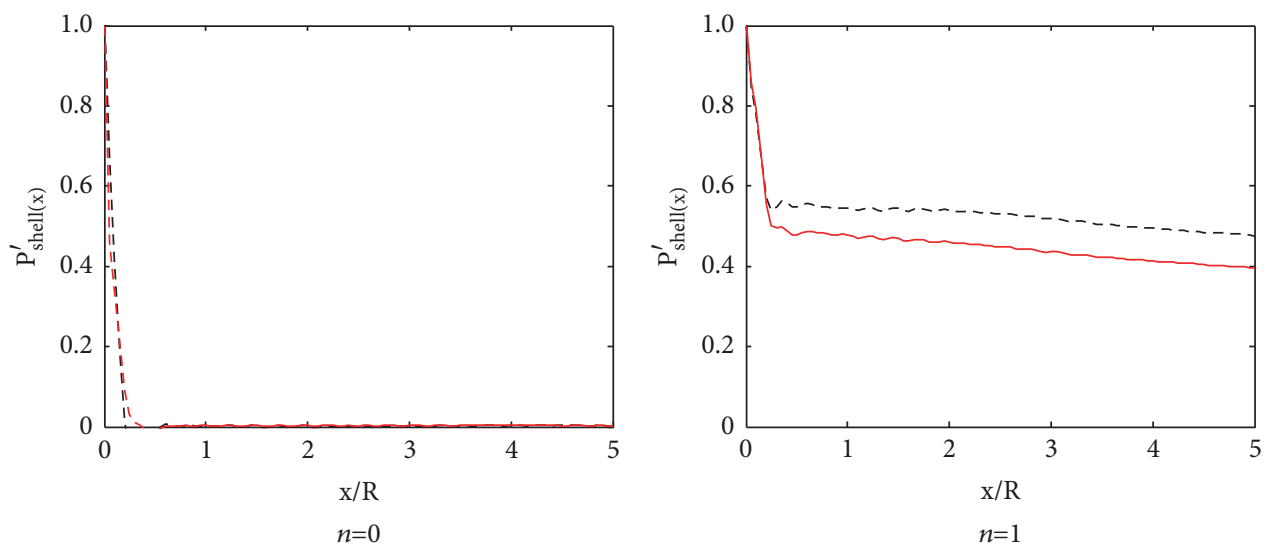

(a)
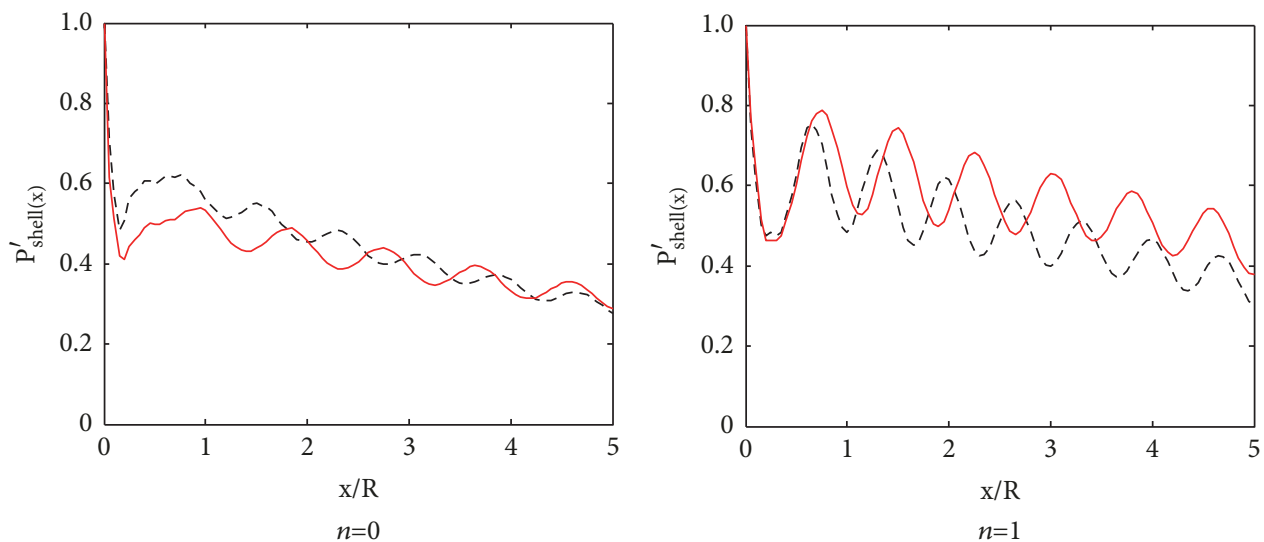

(b)
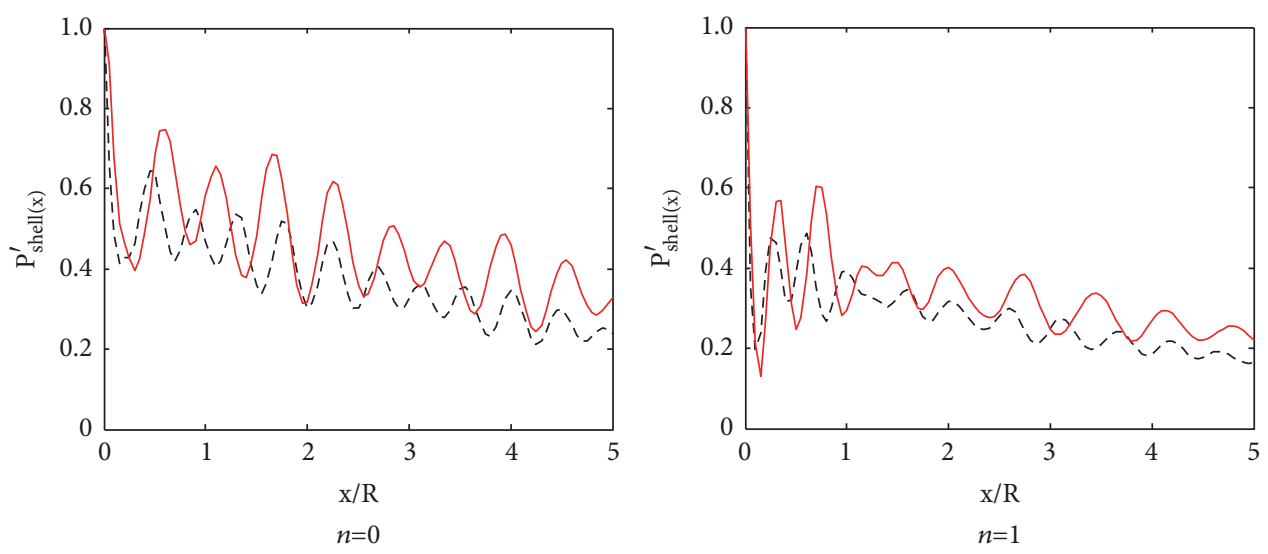

(c)

Figure 7: The comparison of power transmitted by the shell with different cross-ply: (a) $\Omega=0.3$, (b) $\Omega=1.2$, and (c) $\Omega=3.0$; black dashed line: $\left[90^{\circ} / 0^{\circ} / 90^{\circ}\right]$; red solid line: $\left[0^{\circ} / 90^{\circ} / 0^{\circ}\right]$.

ratio of axial stiffness to circumferential stiffness of composite cylindrical shell decreases relatively, so that the input power flow into shell increases.

The power flow transmitted by internal forces (moments) of the shell with cross-ply $\left[90^{\circ} / 0^{\circ} / 90^{\circ}\right]$ against the axial distance $x / R$ is shown in Figure 6. The characteristics of the propagation power flow for cross-ply $\left[90^{\circ} / 0^{\circ} / 90^{\circ}\right]$ shell are similar to those of cross-ply $\left[0^{\circ} / 90^{\circ} / 0^{\circ}\right]$ shell. It is obvious that the power flow carried by different shell forces (moments) is periodic function of distance $x / R$ at middle frequencies and high frequencies. As the frequencies increase, the vibration period becomes much shorter due to the effect of coupling between the shell and fluid for two cases. This indicates that the layer orientation of fiber has little influence on the power flow transmitted by internal forces.

The power flow $P_{\text {shell }(x)}^{\prime}$ transmitted by the shell with cross-ply $\left[90^{\circ} / 0^{\circ} / 90^{\circ}\right]$ in the coupled system is described in Figure 7 . The attenuation of the cross-ply $\left[90^{\circ} / 0^{\circ} / 90^{\circ}\right]$ shell 

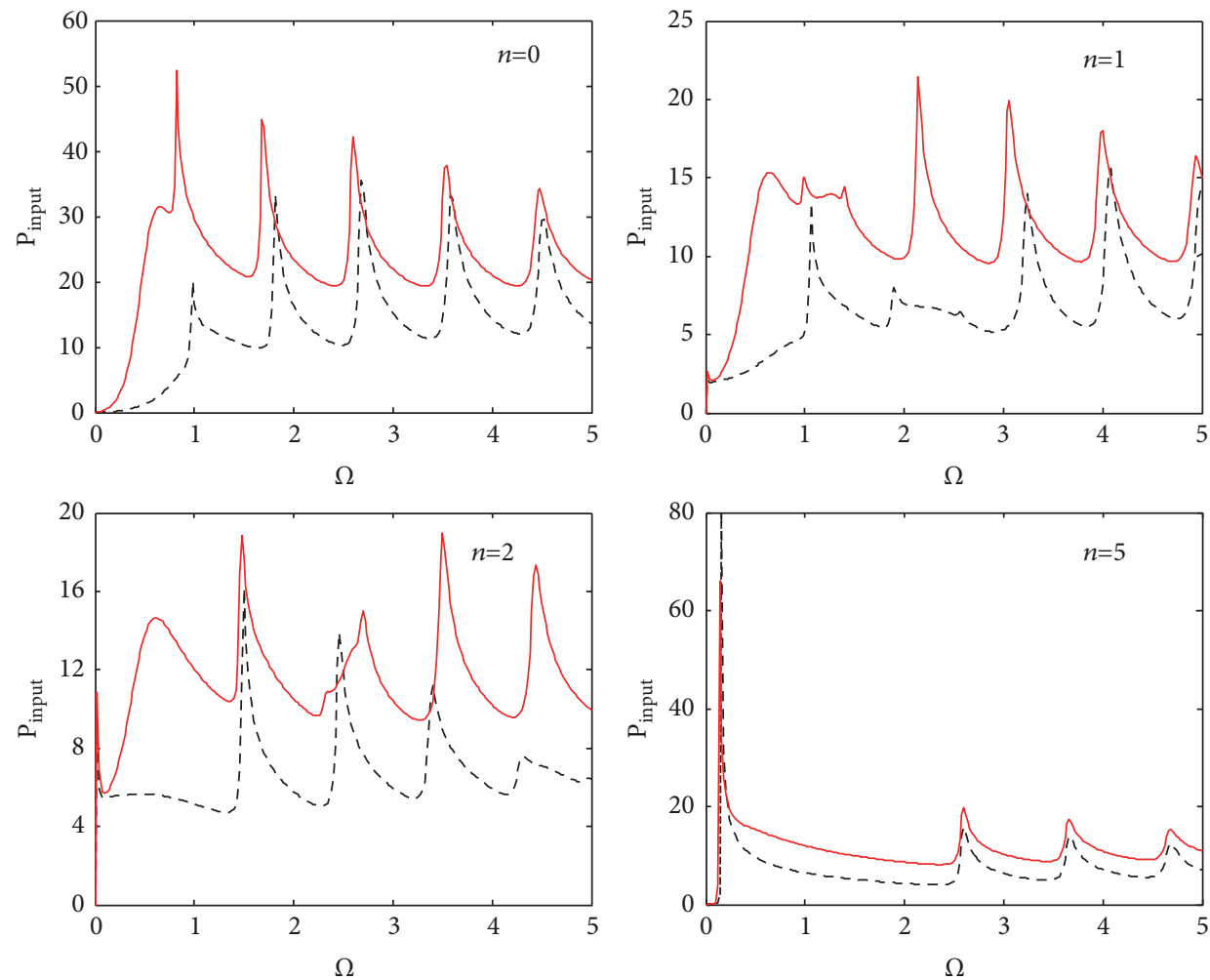

FIGURE 8: The comparison of nondimensional input power flow into the shell with $E_{11} / E_{22}=15$; black dashed line: $E_{11} / E_{22}=15$; red solid line: $E_{11} / E_{22}=2.5$.

is similar to that in the cross-ply $\left[0^{\circ} / 90^{\circ} / 0^{\circ}\right]$ shell. At low frequencies, the curve of power transmitted by the shell is nearly linear. This implies that the effect of shell-fluid coupling at low frequencies is weak so that wave motion phenomena can hardly be observed. With the increasing of frequencies, wave motion phenomena can be obviously found because of the strong effect of shell-fluid coupling. Compared with the cross-ply $\left[0^{\circ} / 90^{\circ} / 0^{\circ}\right]$ shell, power transmitted by the shell for the cross-ply $\left[90^{\circ} / 0^{\circ} / 90^{\circ}\right]$ shell is slightly stronger at middle and high frequencies and weaker at low frequencies, but the difference is relatively small. This indicates that the cross-ply has little influence on the power transmitted by the shell at high frequencies.

6.3. The Influence of Modulus Ratio $E_{11} / E_{22}$ of Material. In order to investigate the influence of modulus ratio $E_{11} / E_{22}$ on the vibration analysis of the coupled system, the material stiffness increases to $E_{11} / E_{22}=15$, six times that in the original model. The input power flow against the driving frequency for fluid-filled cylindrical shell is presented in Figure 8. It is obvious that the wave amplitudes of the power transmitted by the shell decrease significantly. According to the numerical results, it shows that the power flow into the shell decreases substantially as the axial stiffness increases. So, from the viewpoint of vibration control, it seems useful to properly increase the axial stiffness of composite cylindrical shell.

The power flow transmitted by internal forces (moments) of the thicker shell against the axial distance $x / R$ is shown in Figure 9. Compared with the results of original model, the variable amplitudes become much more intense at corresponding frequencies for any circumferential mode order. This indicates that the effect of coupling between the shell and fluid becomes intense as the material stiffness $E_{11} / E_{22}$ increases. For circumferential mode order $n=0$, the power flow $P_{M(x)}^{\prime}$ carried by bending moment increases as the frequency increases, while the power flow $P_{S(x)}^{\prime}$ carried by transverse shear force increases as the frequency increases for circumferential mode order $n=1$. This implies that the torsional motion strengthens as the frequency increases for circumferential mode order $n=0$, while the flexural motion enhances as the frequency increases for circumferential mode order $n=1$.

The power flow $P_{\text {shell }(x)}^{\prime}$ transmitted by the shell in the coupled system is described in Figure 10. For circumferential mode order $n=0$, the power transmitted by the shell rapidly decreases to zero at low frequencies, while the power transmitted by the shell with $E_{11} / E_{22}=15$ is bigger than that of original model. At middle and high frequencies, the power transmitted by the shell decreases rapidly and the wave motion phenomena become particularly intense due to the effect of shell-fluid coupling becoming stronger. This is because of the increasing of $E_{11} / E_{22}$, which makes the vibrational wave pattern more complex and enhances the coupling effects. Thus, the ability to control the input power flow into the coupled system is improved as axial stiffness strengthens. This indicates that increasing the axial stiffness in the cylindrical shell can greatly improve the performance of the coupled system. 

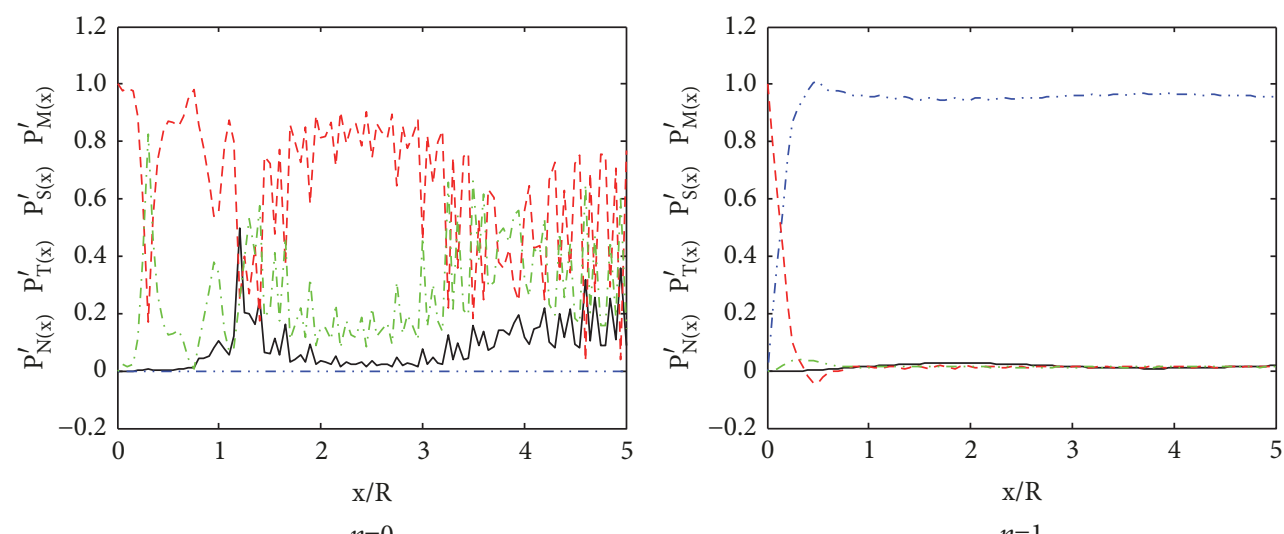

(a)
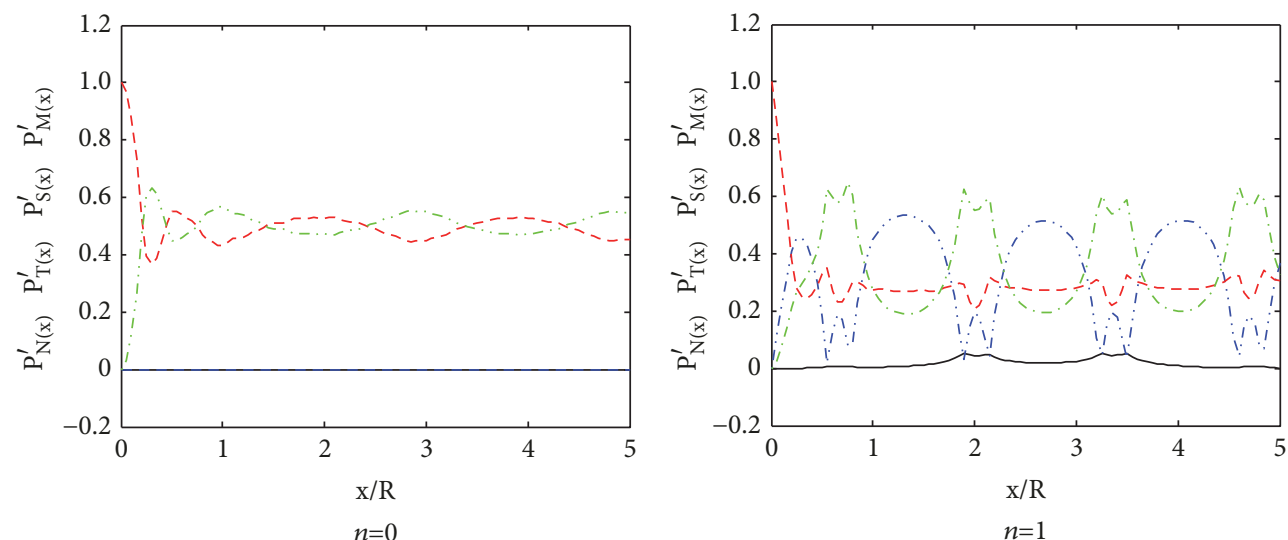

(b)
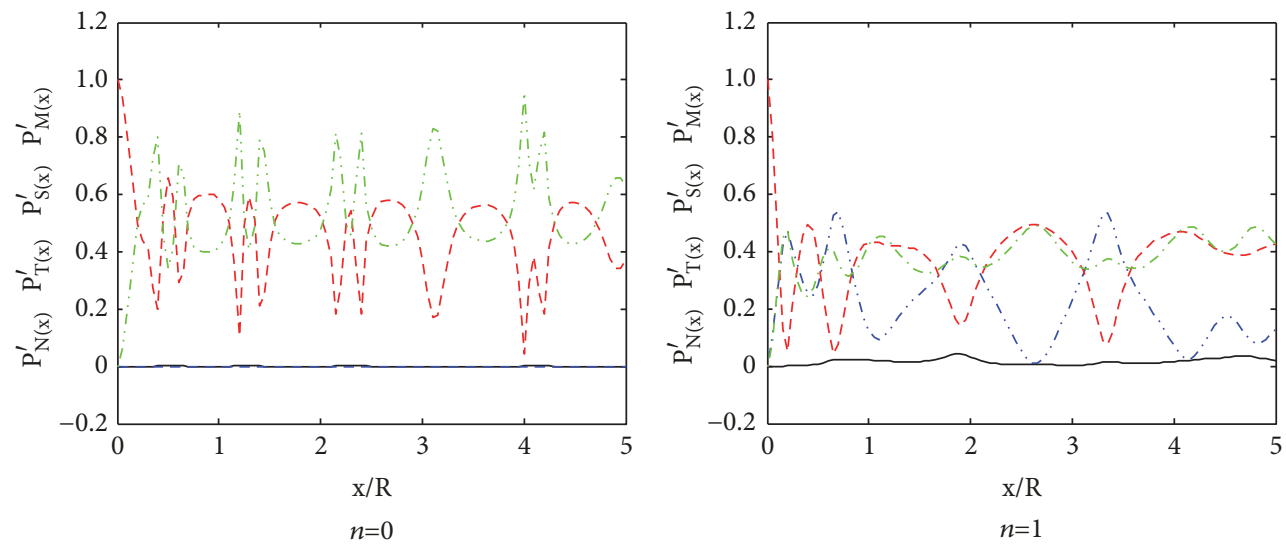

(c)

Figure 9: Power transmitted by different shell forces (moments) with $E_{2} / E_{1}=15$ : (a) $\Omega=0.3$, (b) $\Omega=1.2$, and (c) $\Omega=3.0$; black solid line: $P_{S(x)}^{\prime}$; blue dashed double dotted line: $P_{T(x)}^{\prime}$; red dashed line: $P_{S(x)}^{\prime}$; green dashed dotted line: $P_{M(x)}^{\prime}$.

6.4. The Influence of Thickness-to-Radius Parameter $h / R$. In order to investigate the influence of shell thickness-toradius on the vibration analysis of the coupled system, the shell thickness increases to $h / R=0.075$ with cross-ply $\left[0^{\circ} / 90^{\circ} / 0^{\circ}\right]_{\mathrm{s}}$, twice that in the original model. The input flow against the driving frequency for fluid-filled cylindrical thicker shell is plotted in Figure 11. From the results, it can be seen that the power flow transmitted by the shell along the whole frequency bands reduced remarkably with the increasing of shell thickness. This indicates that the shell thickness has huge effect on the power flow into the shell. 

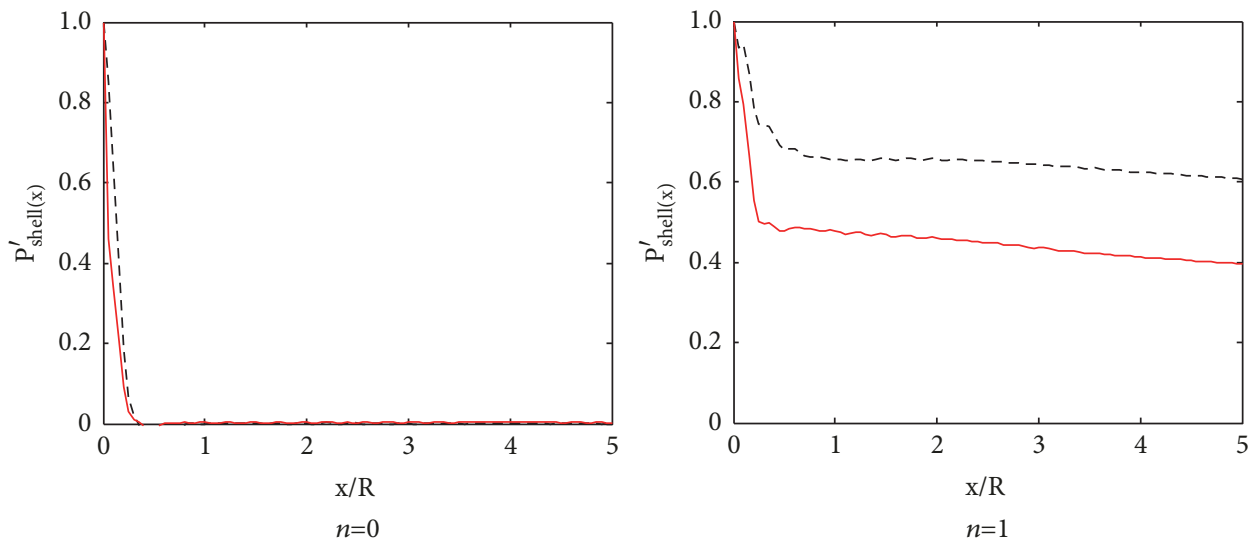

(a)
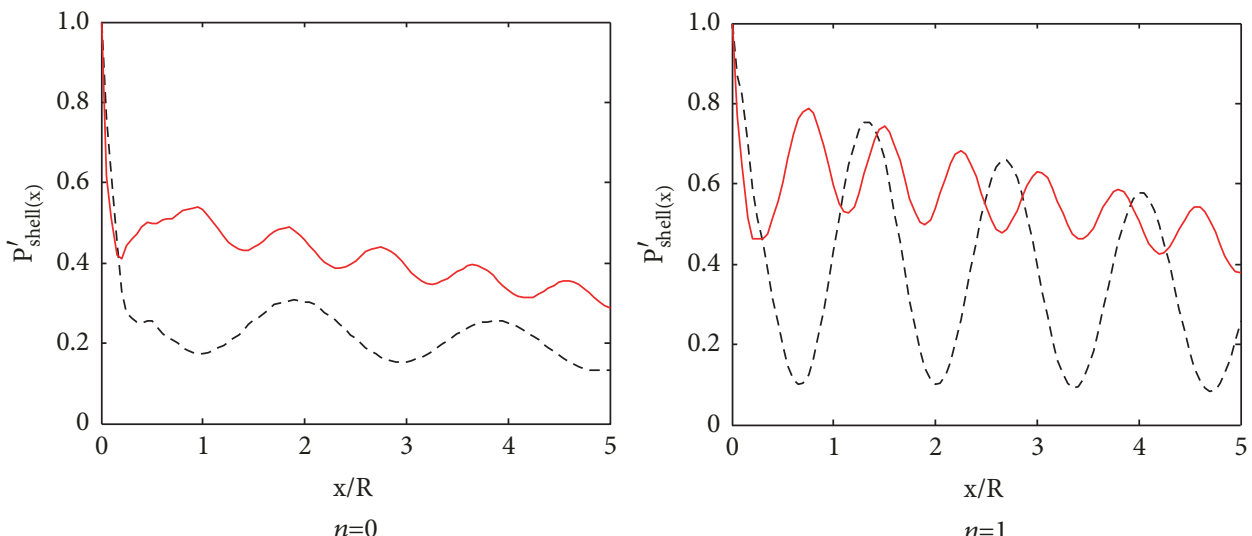

(b)
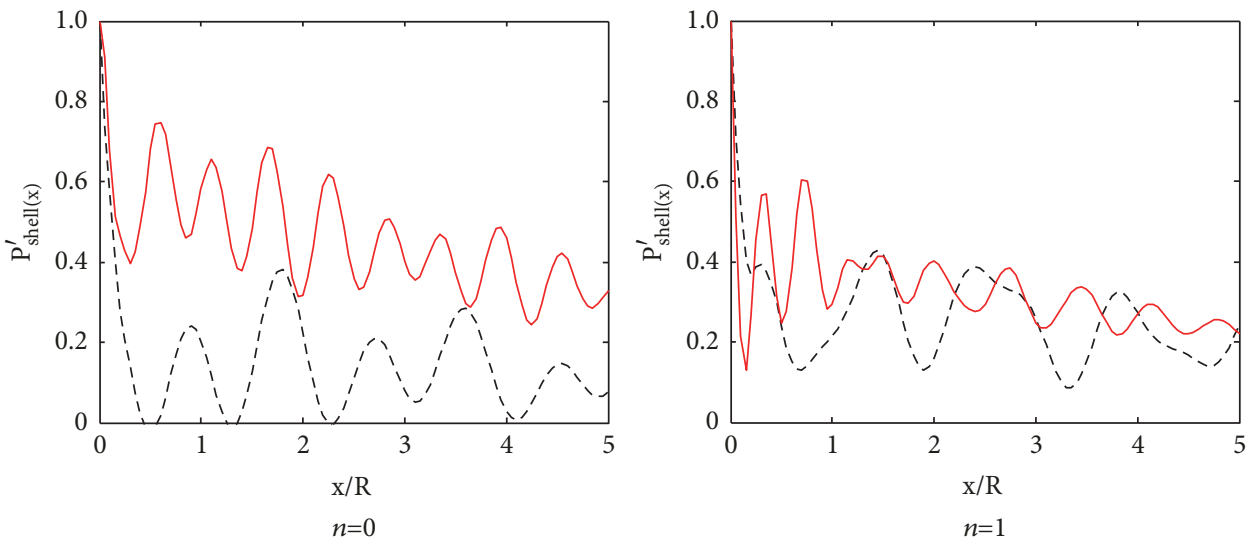

(c)

FIGURE 10: The comparison of power transmitted by the shell with $E_{2} / E_{1}=15$ : (a) $\Omega=0.3$, (b) $\Omega=1.2$, and (c) $\Omega=3.0$; black dashed line: $E_{11} / E_{22}=15$; red solid line: $E_{11} / E_{22}=2.5$.

The power flow transmitted by internal forces (moments) of the thicker shell against the axial distance $x / R$ is displayed in Figure 12. For circumferential mode order $n=0, P_{S(x)}^{\prime}+$ $P_{M(x)}^{\prime}>P_{N(x)}^{\prime}+P_{T(x)}^{\prime}$, the shell motion is predominantly in the radial direction. When the circumferential mode order $n=1$, the torsional motion is coupled with other motions; apart from the close range of the driving point, the torsional motion of the shell wall is primarily the wave motion mode at low frequency. As the frequency increases, the dominating motion gradually attenuates while other motion modes gradually increase, especially for extensional motion along the axial direction. Besides, it is obvious that the amplitude of the power flow transmitted by different internal forces increases as the frequency increases for any circumferential mode order.

The power flow $P_{\text {shell }(x)}^{\prime}$ transmitted by the thicker shell in the coupled system is described in Figure 13. Compared with the original model, the attenuation mode of the thicker 

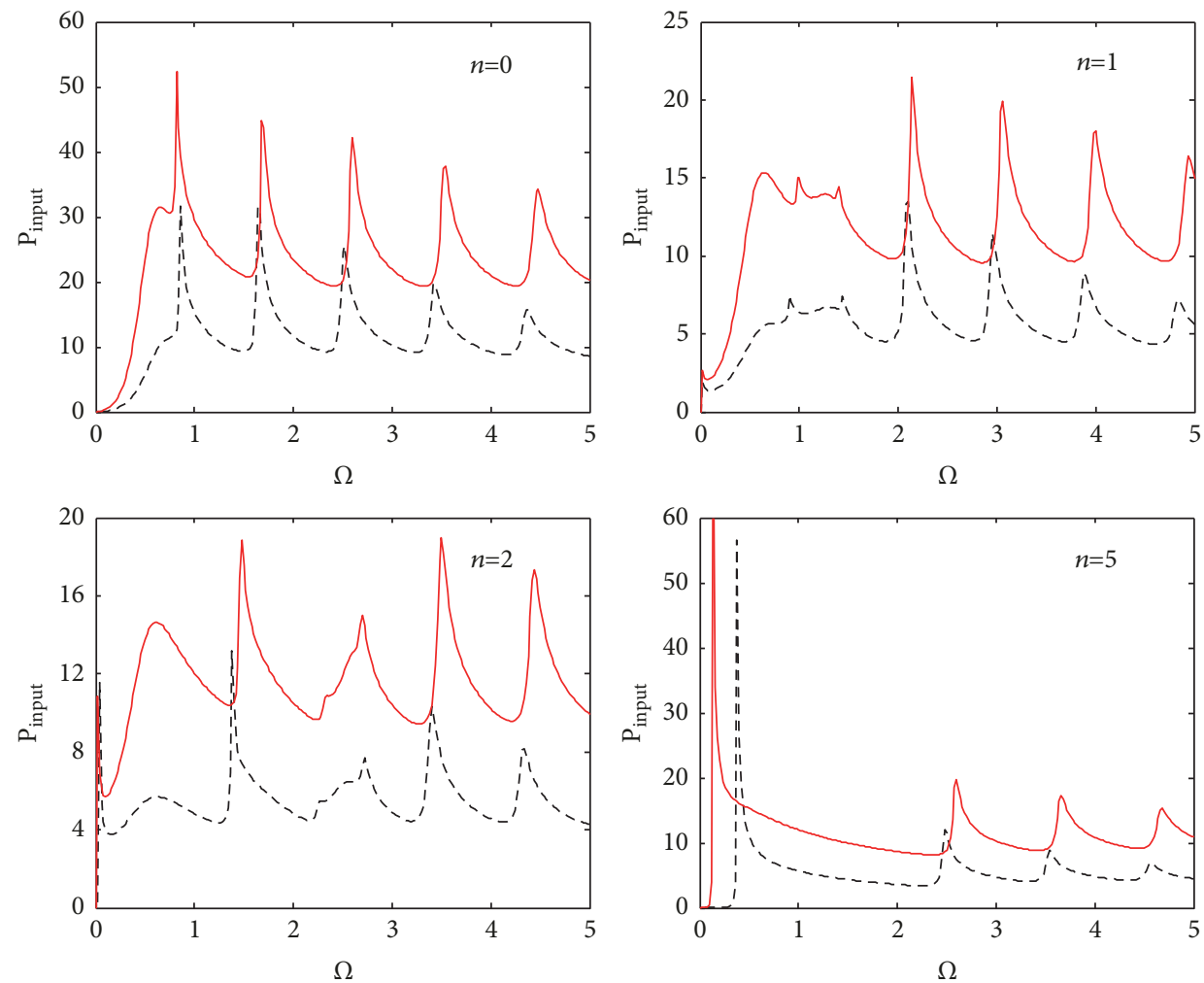

FIGURE 11: The comparison of nondimensional input power flow into the shell with $h / R=0.004$; black dashed line: $h / R=0.075$; red solid line: $h / R=0.0375$.

shell exhibits intense periodic wave motion, which means there is a strong shell-fluid coupling for the thicker shell. At low frequencies, there is no obvious fluctuation for the attenuation of the power transmitted by the shell. This indicates that the effect of shell-fluid coupling is weak. For circumferential mode order $n=0$, the power transmitted by the shell rapidly decreases to zero at low frequencies, while the power transmitted by the thicker shell is bigger than that of original model. At middle frequencies, the magnitude of power transmitted by the thicker shell is almost the same as that of the original model, whereas the amplitude of the resonance hump is further enlarged. At high frequencies, the power transmitted by the thicker shell decreases rapidly and the wave motion phenomena become particularly intense due to the effect of shell-fluid coupling becoming stronger. This indicates that the thickness of shell has a strong influence on the vibrational power flow propagation. So, making use of thicker shell in middle and high frequency bands will be helpful to control structure vibration.

\section{Conclusions}

The paper aims to investigate the characteristics of vibrational power flow in an infinite laminated composite cylindrical shell filled with fluid by using the wave propagation approach. The vibrational power flow input into the coupled system and its propagation are established under a circumferential line cosine harmonic force. Numerical computations are implemented to study the effects of the fiber orientation, modulus ratio $E_{11} / E_{22}$, and thickness-to-radius parameter $h / R$ on input power into the coupled system and propagation power along the shell axial direction. Based on the numerical results, the following conclusions can be drawn:

(1) The wave propagation approach is a noniterative, less computationally intensive, and reasonably accurate method. It can easily handle the vibration analysis of composite shells.

(2) Filled water in the shell will greatly influence the input power flow due to an additional mass of water and the shell-fluid interaction at middle frequencies. The propagation power carried by the transverse shear force is almost equal to that carried by the bending moments at middle frequencies and high frequencies for any circumferential mode order.

(3) The input power flow into shell with cross-ply $\left[90^{\circ} / 0^{\circ} / 90^{\circ}\right]$ is larger than that into shell with cross-ply $\left[0^{\circ} / 90^{\circ} / 0^{\circ}\right]$, while the layer orientation of fiber has little influence on the power flow transmitted by internal forces.

(4) The power flow into the shell decreases substantially as the modulus ratio $E_{11} / E_{22}$ increases, so increasing the axial stiffness in the cylindrical shell can greatly improve the performance of the coupled system.

(5) The thickness of shell has a strong influence on the vibrational power flow propagation; thus, making use of thicker shell in middle and high frequency bands will be helpful to control structure vibration. 

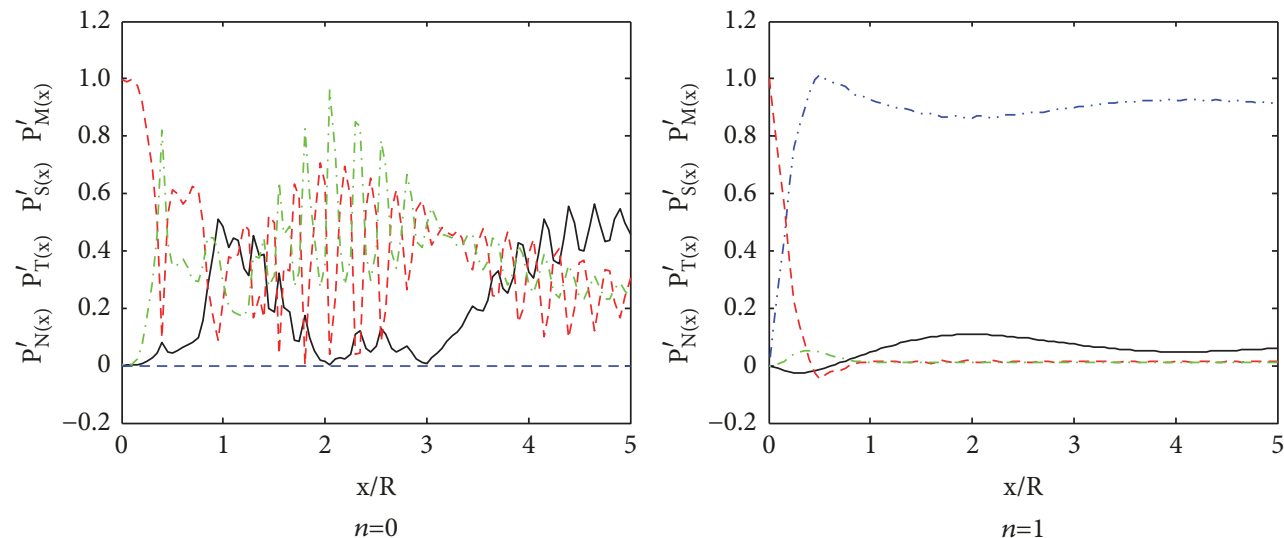

(a)
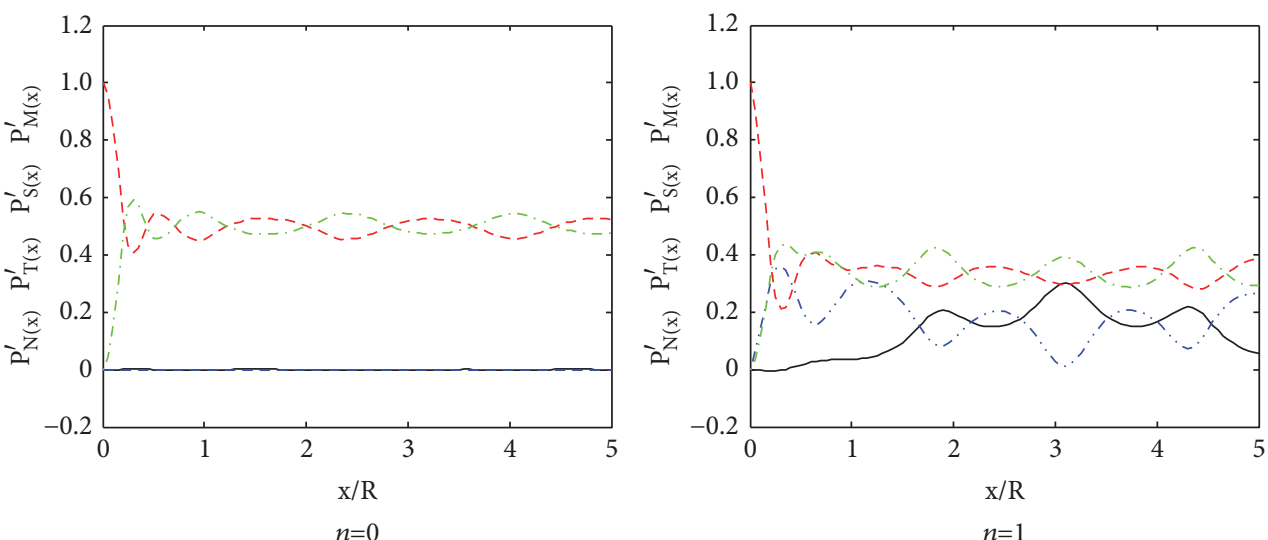

(b)
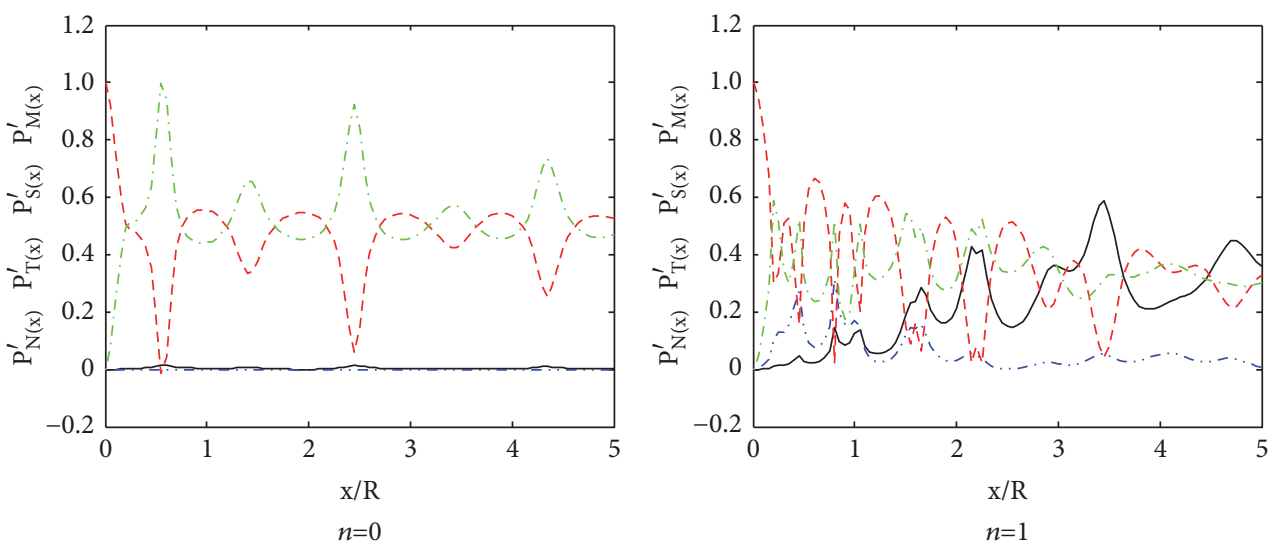

(c)

FiguRE 12: Power transmitted by different shell forces (moments) with $h / R=0.075$ : (a) $\Omega=0.3$, (b) $\Omega=1.2$, and (c) $\Omega=3.0$; black solid line: $P_{S(x)}^{\prime}$; blue dashed double dotted line: $P_{T(x)}^{\prime}$; red dashed line: $P_{S(x)}^{\prime}$; green dashed dotted line: $P_{M(x)}^{\prime}$.

\section{Conflicts of Interest}

The authors declare that there are no conflicts of interest regarding the publication of this paper.

\section{Acknowledgments}

The present work is supported by the National Natural Science Foundation of China (Grants nos. 51609089, 51579110, and 51079059), the China Postdoctoral Science Foundation (Grant no. 2016M592338), the National High Technology Research and Development Program of China (863 Program, Grant no. 2012AA112601), the Offshore Flexible Pipe Project from Ministry of Industry and Information Technology, and the Fund Project Independent Innovation Research Fund of Huazhong University of Science and Technology (Grant no. 2015TS004). 

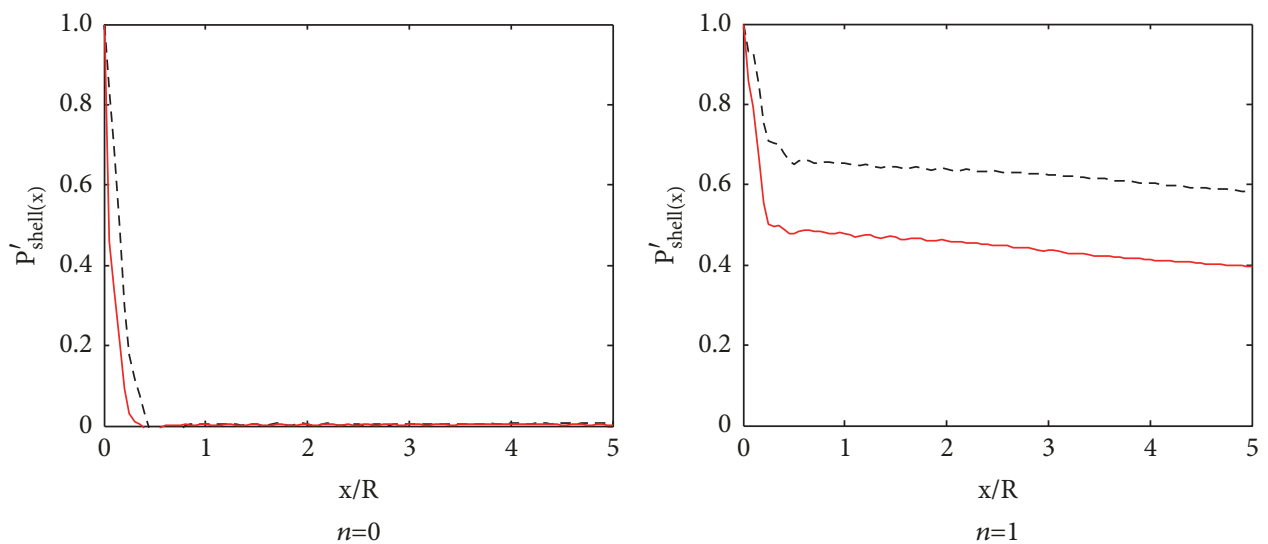

(a)
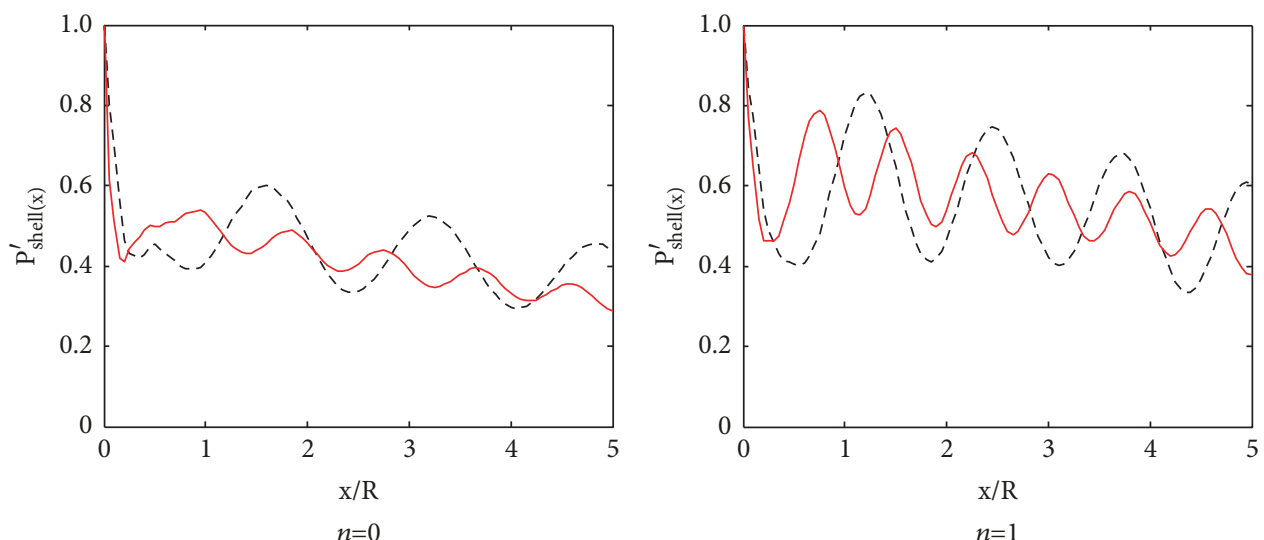

(b)
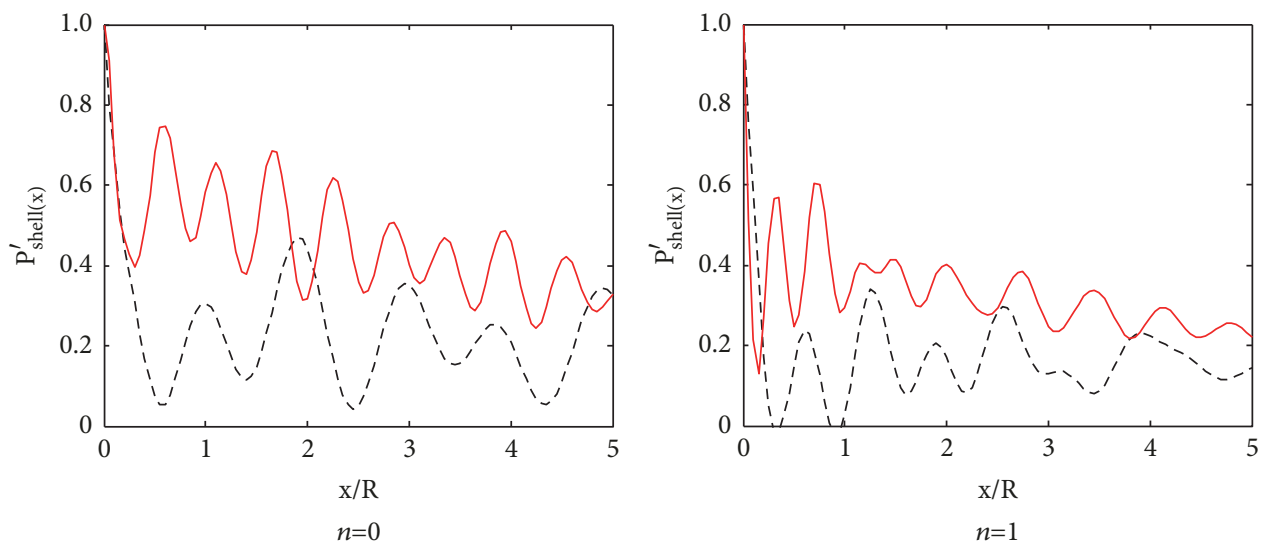

(c)

Figure 13: The comparison of power transmitted by the shell with $h / R=0.075$ : (a) $\Omega=0.3$, (b) $\Omega=1.2$, and (c) $\Omega=3.0$; black dashed line: $h / R=0.075$; red solid line: $h / R=0.0375$.

\section{References}

[1] Y. Luo, T. Qu, B. Zhang, Y. Pan, and P. Xiao, "Simulations and Experiments on the Vibrational Characteristics of Cylindrical Shell Resonator Actuated by Piezoelectric Electrodes with Different Thicknesses," Shock and Vibration, vol. 2017, Article ID 2314858, 2017.

[2] W. He, J. Liu, B. Tao, D. Xie, J. Liu, and M. Zhang, "Experimental and numerical research on the low velocity impact behavior of hybrid corrugated core sandwich structures," Composite Structures, vol. 158, pp. 30-43, 2016.

[3] H.-L. Dai and H.-J. Jiang, "Forced vibration analysis for a FGPM cylindrical shell," Shock and Vibration, vol. 20, no. 3, pp. 531-550, 2013.

[4] J. Liu, W. He, D. Xie, and B. Tao, “The effect of impactor shape on the low-velocity impact behavior of hybrid corrugated core sandwich structures," Composites Part B: Engineering, vol. 111, pp. 315-331, 2017. 
[5] D. Shi, S. Zha, H. Zhang, and Q. Wang, "Free Vibration Analysis of the Unified Functionally Graded Shallow Shell with General Boundary Conditions," Shock and Vibration, vol. 2017, Article ID 7025190, 2017.

[6] Q. Tang, C. Li, and B. Wen, "Analysis on Forced Vibration of Thin-Wall Cylindrical Shell with Nonlinear Boundary Condition," Shock and Vibration, vol. 2016, Article ID 8978932, 2016.

[7] D. Tang, G. Wu, X. Yao, and C. Wang, "Free Vibration Analysis of Circular Cylindrical Shells with Arbitrary Boundary Conditions by the Method of Reverberation-Ray Matrix," Shock and Vibration, vol. 2016, Article ID 3814693, 2016.

[8] K. Y. Lam and W. Qian, "Free vibration of symmetric angle-ply thick laminated composite cylindrical shells," Composites Part B: Engineering, vol. 31, no. 4, pp. 345-354, 2000.

[9] R.-C. Shi, R.-Y. Huang, G.-Y. Wang, Y.-K. Wang, and Y.-C. Li, "Numerical simulation of underwater shock wave propagation in the vicinity of rigid wall based on ghost fluid method," Shock and Vibration, vol. 2015, Article ID 467376, 16 pages, 2015.

[10] M. Chen, J. Wei, K. Xie, N. Deng, and G. Hou, "Wave based method for free vibration analysis of ring stiffened cylindrical shell with intermediate large frame ribs," Shock and Vibration, vol. 20, no. 3, pp. 459-479, 2013.

[11] K. Y. Lam and C. T. Loy, "Influence of boundary conditions and fibre orientation on the natural frequencies of thin orthotropic laminated cylindrical shells," Composite Structures, vol. 31, no. 1, pp. 21-30, 1995.

[12] A. A. Jafari, S. M. R. Khalili, and R. Azarafza, “Transient dynamic response of composite circular cylindrical shells under radial impulse load and axial compressive loads," Thin-Walled Structures, vol. 43, no. 11, pp. 1763-1786, 2005.

[13] A. J. M. Ferreira, C. M. C. Roque, and R. M. N. Jorge, "Natural frequencies of FSDT cross-ply composite shells by multiquadrics," Composite Structures, vol. 77, no. 3, pp. 296-305, 2007.

[14] A. Alibeigloo, "Static and vibration analysis of axi-symmetric angle-ply laminated cylindrical shell using state space differential quadrature method," International Journal of Pressure Vessels and Piping, vol. 86, no. 11, pp. 738-747, 2009.

[15] E. Asadi, W. Wang, and M. S. Qatu, "Static and vibration analyses of thick deep laminated cylindrical shells using 3D and various shear deformation theories," Composite Structures, vol. 94, no. 2, pp. 494-500, 2012.

[16] E. Viola, F. Tornabene, and N. Fantuzzi, "General higher-order shear deformation theories for the free vibration analysis of completely doubly-curved laminated shells and panels," Composite Structures, vol. 95, pp. 639-666, 2013.

[17] S. M. R. Khalili, R. Azarafza, and A. Davar, "Transient dynamic response of initially stressed composite circular cylindrical shells under radial impulse load," Composite Structures, vol. 89, no. 2, pp. 275-284, 2009.

[18] M. Amabili and J. N. Reddy, "A new non-linear higher-order shear deformation theory for large-amplitude vibrations of laminated doubly curved shells," International Journal of NonLinear Mechanics, vol. 45, no. 4, pp. 409-418, 2010.

[19] X. M. Zhang, G. R. Liu, and K. Y. Lam, "Coupled vibration analysis of fluid-filled cylindrical shells using the wave propagation approach," Applied Acoustics, vol. 62, no. 3, pp. 229-243, 2001.

[20] Z. Iqbal, M. N. Naeem, N. Sultana, S. H. Arshad, and A. G. Shah, "Vibration characteristics of FGM circular cylindrical shells filled with fluid using wave propagation approach," Applied Mathematics and Mechanics-English Edition, vol. 30, no. 11, pp. 1393-1404, 2009.
[21] T. Natsuki, Q.-Q. Ni, and M. Endo, "Vibrational analysis of fluid-filled carbon nanotubes using the wave propagation approach," Applied Physics A: Materials Science \& Processing, vol. 90, no. 3, pp. 441-445, 2008.

[22] R. Kadoli and N. Ganesan, "Free vibration and buckling analysis of composite cylindrical shells conveying hot fluid," Composite Structures, vol. 60, no. 1, pp. 19-32, 2003.

[23] M. Paak, M. P. Païdoussis, and A. K. Misra, "Nonlinear vibrations of cantilevered circular cylindrical shells in contact with quiescent fluid," Journal of Fluids and Structures, vol. 49, pp. 283-302, 2014.

[24] Z. C. Xi, L. H. Yam, and T. P. Leung, "Free vibration of a laminated composite circular cylindrical shell partially filled with fluid," Composites Part B: Engineering, vol. 28, no. 4, pp. 359375, 1997.

[25] M. R. Saviz, "Coupled vibration of partially fluid-filled laminated composite cylindrical shells," Journal of Solid Mechanics, vol. 8, no. 4, pp. 823-839, 2016.

[26] M. B. Xu and W. H. Zhang, "Vibrational power flow input and transmission in a circular cylindrical shell filled with fluid," Journal of Sound and Vibration, vol. 234, no. 3, pp. 387-403, 2000.

[27] J. Yan, T. Y. Li, J. X. Liu, and X. Zhu, "Space harmonic analysis of sound radiation from a submerged periodic ring-stiffened cylindrical shell," Applied Acoustics, vol. 67, no. 8, pp. 743-755, 2006.

[28] L. Gan, X. B. Li, and Z. Zhang, "Free vibration analysis of ringstiffened cylindrical shells using wave propagation approach," Journal of Sound and Vibration, vol. 326, no. 3-5, pp. 633-646, 2009.

[29] A. Bahrami and A. Teimourian, "Study on the effect of small scale on the wave reflection in carbon nanotubes using nonlocal Timoshenko beam theory and wave propagation approach," Composites Part B: Engineering, vol. 91, pp. 492-504, 2016.

[30] K. Y. Lam and C. T. Loy, "Analysis of rotating laminated cylindrical shells by different thin shell theories," Journal of Sound and Vibration, vol. 186, no. 1, pp. 23-35, 1995.

[31] X. M. Zhang, "Vibration analysis of cross-ply laminated composite cylindrical shells using the wave propagation approach," Applied Acoustics, vol. 62, no. 11, pp. 1221-1228, 2001. 


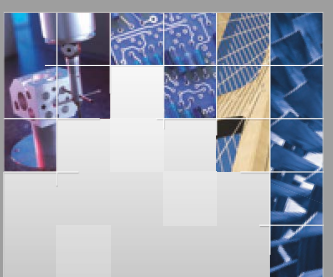

\section{Enfincering}
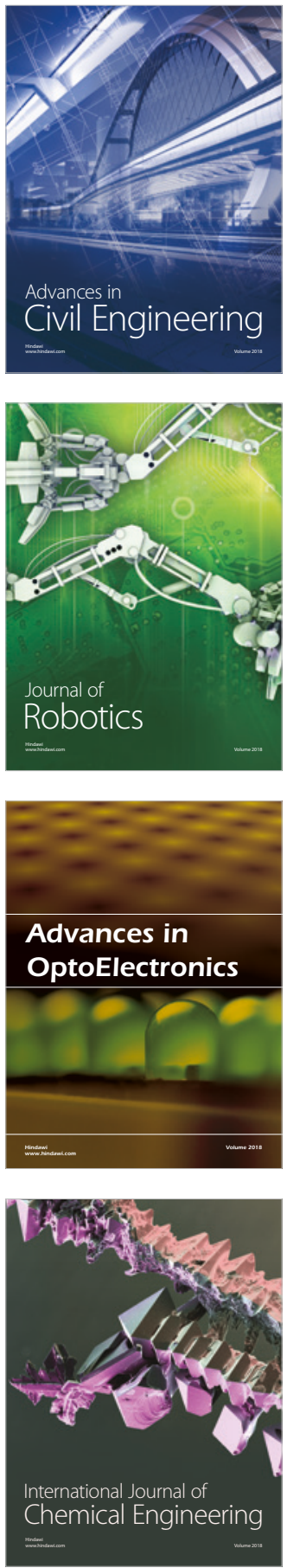

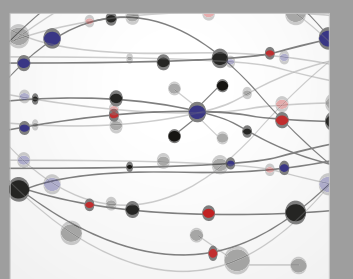

\section{Rotating \\ Machinery}

The Scientific World Journal

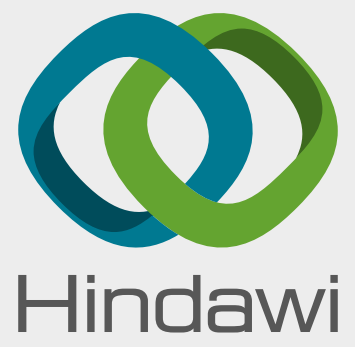

Submit your manuscripts at

www.hindawi.com
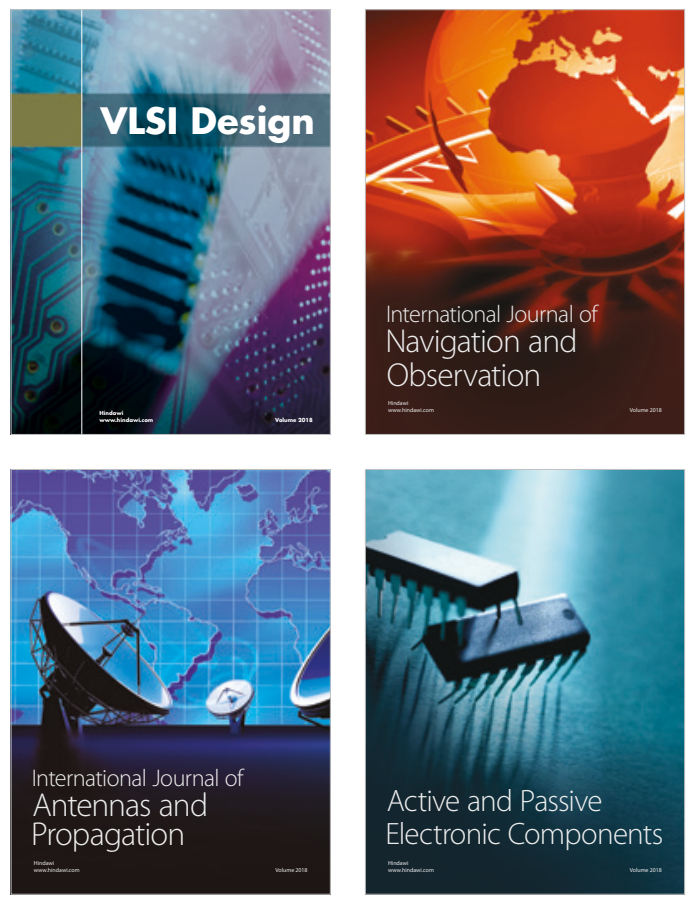
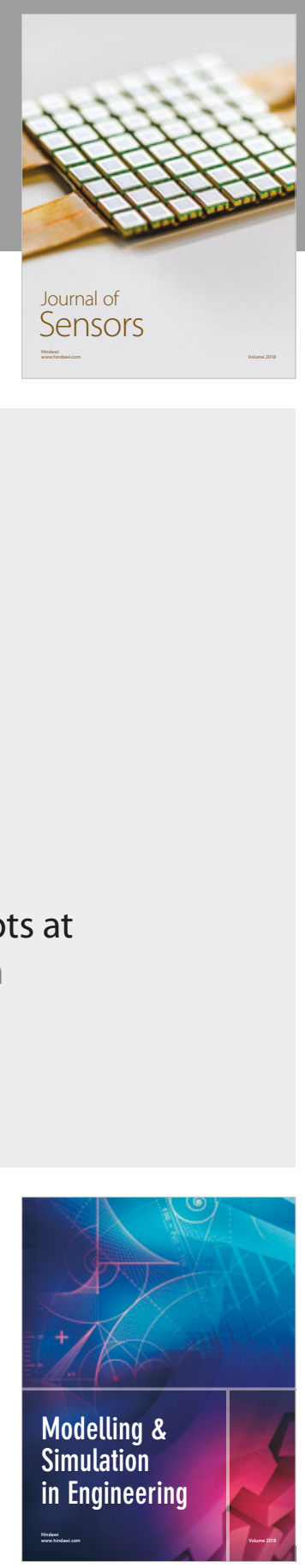

\section{Advances \\ Multimedia}
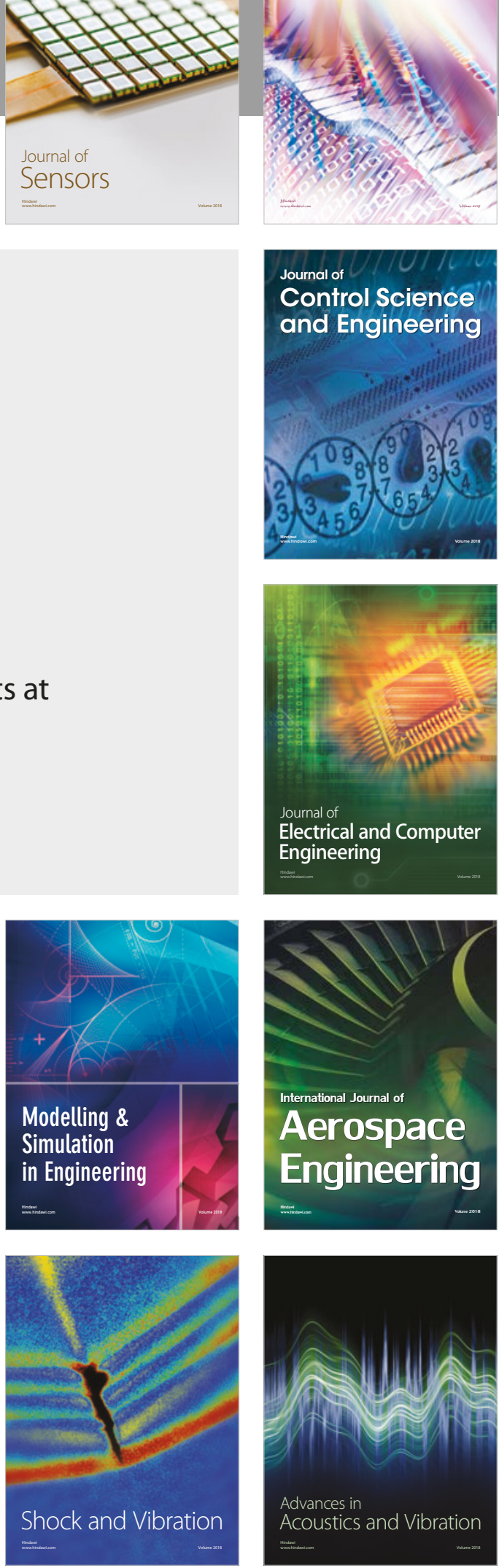